\title{
Stability in Mean of Partial Variables for Coupled Stochastic Reaction-Diffusion Systems on Networks: A Graph Approach
}

\author{
Yonggui Kao ${ }^{1}$ and Hamid Reza Karimi ${ }^{2}$ \\ ${ }^{1}$ Department of Mathematics, Harbin Institute of Technology, Weihai 264209, China \\ ${ }^{2}$ Department of Engineering, Faculty of Technology and Science, University of Agder, 4898 Grimstad, Norway \\ Correspondence should be addressed to Hamid Reza Karimi; hamid.r.karimi@uia.no
}

Received 24 February 2014; Accepted 11 April 2014; Published 7 May 2014

Academic Editor: Jun $\mathrm{Hu}$

Copyright (c) 2014 Y. Kao and H. R. Karimi. This is an open access article distributed under the Creative Commons Attribution License, which permits unrestricted use, distribution, and reproduction in any medium, provided the original work is properly cited.

\begin{abstract}
This paper is devoted to investigating stability in mean of partial variables for coupled stochastic reaction-diffusion systems on networks (CSRDSNs). By transforming the integral of the trajectory with respect to spatial variables as the solution of the stochastic ordinary differential equations (SODE) and using Itô formula, we establish some novel stability principles for uniform stability in mean, asymptotic stability in mean, uniformly asymptotic stability in mean, and exponential stability in mean of partial variables for CSRDSNs. These stability principles have a close relation with the topology property of the network. We also provide a systematic method for constructing global Lyapunov function for these CSRDSNs by using graph theory. The new method can help to analyze the dynamics of complex networks. An example is presented to illustrate the effectiveness and efficiency of the obtained results.
\end{abstract}

\section{Introduction}

Coupled systems on networks (CSNs), composed of a large number of highly interconnected dynamical nodes [1], have received more and more attention due to its popularity in modelling many large-scale dynamical systems from science and engineering, such as communication networks, social networks, power grids, cellular networks, World Wide Web, metabolic systems, food webs, and disease transmission networks; see for instance [2-6] and the references therein. Stability is one important constituent part of performance investigation for dynamical systems, and it is very necessary to construct a relation between the stability criteria of a CSN and some topology properties of the network [7-11]. $\mathrm{Li}$ and Shuai [11] have considered global stability for the general CSNs based on graph theory, without discussing the stochastic effects. Due to the fact that most motions are actually the results of deterministic processes mingling with random processes [12, 13], Kao et al. [14] have investigated stability of coupled stochastic systems with time-delay on networks without reaction diffusion effects. In fact, for many realistic CSNs, the node state is seriously dependent on the time and space [15-20]. Hence, in order to describe more accurately the dynamics changes of CSNs, Kao and Wang put up with stochastic coupled reaction-diffusion systems on networks (SCEDSNs) based on graph theory and probed global stability analysis for SCEDSNs [21].

On the other hand, in real world, it is difficult or even impossible to measure or estimate all the states of the systems due to the factors of expensive cost or technique [22-26]. Partial stability technique (stability of part of the variables) is most useful when a fully stabilized system losses some control engines or some phase variables are not actively controlled. Such situations are most applicable for automatic systems which need to work remotely without a proper access to maintenance, such as satellite or robots. Therefore, stability and stabilization of motion with respect to part of the variables is of great significance [27-38]. Kao et al. [27] have studied stability in mean of partial variables for stochastic reaction-diffusion systems with Markovian switching. Xi et al. [31] have investigated output consensus analysis and design for high-order linear swarm systems by partial stability method. Partial stabilization technology has been applied into the guidance problem by Shafiei and 
Binazadeh [32]. Ignatyev [34] has probed partial asymptotic stability in probability of stochastic differential equations. Chen et al. [36] have discussed impulsive synchronization of chaotic Lur'e systems via partial states. Stability in mean of partial variables for stochastic reaction-diffusion systems has been considered in [38]. To the best of the authors' knowledge, stability analysis for stability in mean of partial variables for coupled stochastic reaction-diffusion systems on networks (CSRDSNs) has not been properly addressed, which still remains important and challenging.

Motivated by the above discussions, in this paper, we propose the CSRDSNs model. In Section 2, some preliminaries are presented. In Section 3, some new stability principles for uniform stability in mean, asymptotic stability in mean, uniformly asymptotic stability in mean, and exponential stability in mean of partial variables for CSRDSNs are established. These stability principles have a close relation to the topology property of the network. A systematic method is provided to construct the global Lyapunov function of CSRDSNs by combining graph theory and the Lyapunov second method. The findings show that, if each vertex system has a globally stable equilibrium and possesses a global Lyapunov function $V_{k}$, then the global Lyapunov function for the CSRDSNs can be systematically produced by individual $V_{k}$. An example is provided in Section 4. Section 5 is conclusion. Notations: for convenience, we sometimes write $\mathbf{v}, \mathbf{v}_{\mathbf{k}}$, and $\mathbf{v}_{\mathbf{j}}$ as $\mathbf{v}(t, \mathbf{x})$, $\mathbf{v}_{\mathbf{k}}(t, \mathbf{x})$, and $\mathbf{v}_{\mathbf{j}}(t, \mathbf{x})$, respectively.

\section{Preliminaries}

A general stochastic reaction-diffusion system reads

$$
\begin{gathered}
\mathrm{d} \mathbf{v}(t, \mathbf{x})=\left[\rho_{i}(t) \Delta \mathbf{v}(t, \mathbf{x})+f(t, \mathbf{x}, \mathbf{v}(t, \mathbf{x}))\right] \mathrm{d} t \\
+g(t, \mathbf{x}, \mathbf{v}(t, \mathbf{x})) \mathrm{d} W(t) \\
(t, \mathbf{x}) \in \mathbb{R}_{+} \times G,
\end{gathered}
$$

with boundary condition

$$
\frac{\partial \mathbf{v}(t, \mathbf{x})}{\partial \mathcal{N}}=0, \quad(t, \mathbf{x}) \in \mathbb{R}_{+} \times \partial G,
$$

where $\mathbf{v}=\operatorname{col}(\mathbf{y}, \mathbf{z}) \in \mathbb{R}^{n}, \mathbf{y} \in \mathbb{R}^{m}, \mathbf{z} \in \mathbb{R}^{p}(m+p=n)$, $\Delta \mathbf{v}=\sum_{i=1}^{n}\left(\partial^{2} \mathbf{v} / \partial x_{i}^{2}\right) ;$ denote $\left|\int_{G} \mathbf{v}(\cdot, \mathbf{x}) \mathrm{d} \mathbf{x}\right|$ by $|\mathbf{v}(\cdot, \mathbf{x})|_{G}, G=$ $\{\mathbf{x},|\mathbf{x}|<l<+\infty\} \subset R^{r}$, and both $f \in\left[\mathbb{R}_{+} \times G \times \Gamma, \mathbb{R}^{n}\right]$ and $g \in$ $\left[\mathbb{R}_{+} \times G \times \Gamma, \mathbb{R}^{n \times m}\right]$ are Borel measurable functions; here, $\Gamma=$ $\left\{\mathbf{v},|\mathbf{y}(\cdot, x)|_{G} \leq H=\right.$ const, $\left.|\mathbf{z}(\cdot, \mathbf{x})|_{G}<+\infty\right\} \subset \mathbb{R}^{n},|\cdot|$ stands for vector norm, $W(t)=\left(W_{1}, \ldots, W_{m}\right)^{\top}$ is an $m$-dimension Brown motion with natural flow $\left\{\mathscr{F}_{t}\right\}_{t>0}$ defined on complete probability space $\left(\Omega, \mathscr{F},\left(\mathscr{F}_{t}\right)_{t \in I}, \mathbb{P}\right), \mathscr{N}$ is the normal vector to $\partial G, f(t, \mathbf{x}, 0) \equiv 0$, and $g(t, \mathbf{x}, 0) \equiv 0$.

Throughout this paper, we suppose function $g(t, \mathbf{x}$, $\mathbf{v}(t, \mathbf{x}))$ satisfies integral linear growth condition and $f, g$ meet Lipschitz condition; that is, there exists constant $L>0$ such that

$$
\begin{gathered}
|g(t, \mathbf{x}, \mathbf{v}(t, \mathbf{x}))|_{G} \leq L\left(1+|\mathbf{v}|_{G}\right), \\
\left|g\left(t, \mathbf{x}, \mathbf{v}_{\mathbf{1}}(t, \mathbf{x})\right)-g\left(t, \mathbf{x}, \mathbf{v}_{\mathbf{2}}(t, \mathbf{x})\right)\right|_{G} \leq L\left|\mathbf{v}_{\mathbf{1}}-\mathbf{v}_{\mathbf{2}}\right|_{G}, \\
\left|f\left(t, \mathbf{x}, \mathbf{v}_{\mathbf{1}}(t, \mathbf{x})\right)-f\left(t, \mathbf{x}, \mathbf{v}_{\mathbf{2}}(t, \mathbf{x})\right)\right|_{G} \leq L\left|\mathbf{v}_{\mathbf{1}}-\mathbf{v}_{\mathbf{2}}\right|_{G},
\end{gathered}
$$

where $\mid \mathbf{v}(\cdot, \mathbf{x}))\left.\right|_{G} \triangleq\left|\int_{G} \mathbf{v}(\cdot, \mathbf{x}) \mathrm{d} \mathbf{x}\right|$. The existence of the solution for system (1) can be proved by the common stepwise interactive method and the relevant conclusion can also refer to $[39,40]$.

Before the start of our discussion, we will first introduce some definitions as to stability in mean of partial variables for stochastic reaction-diffusion systems.

Definition 1. The trivial solution of system (1) is said to be stable in mean as to partial variables y if, for $\forall \varepsilon>0, \forall t_{0}>0$, there is $\delta\left(t_{0}, \varepsilon\right)$ such that $\mathscr{E}\left\{\left|\mathbf{y}\left(t, \mathbf{x}, t_{0}, \mathbf{v}_{0}\right)\right|_{G}\right\}<\varepsilon$ holds for $\forall \mathbf{v}_{0} \in S_{\delta}=\left\{\left.\mathbf{v}|| \mathbf{v}(\cdot, \mathbf{x})\right|_{G}<\delta\right\}$.

The trivial solution of system (1) is said to be uniformly stable in mean as to partial variables $\mathbf{y}$ if, for $\forall \varepsilon>0, \forall t_{0}>0$, there is $\delta(\varepsilon)$ such that $\mathscr{E}\left\{\left|\mathbf{y}\left(t, \mathbf{x}, t_{0}, \mathbf{v}_{0}\right)\right|_{G}\right\}<\varepsilon$ holds for $\forall \mathbf{v}_{0} \in$ $S_{\delta}=\left\{\left.\mathbf{v}|| \mathbf{v}(\cdot, \mathbf{x})\right|_{G}<\delta\right\}$.

The trivial solution of system (1) is said to be asymptotically stable in mean as to partial variables $\mathbf{y}$ if, for $\forall \varepsilon>0, \forall t_{0}>0$, there is $\delta\left(t_{0}, \varepsilon\right)$ such that for $\forall \mathbf{v}_{0} \in$ $S_{\delta}=\left\{\left.\mathbf{v}|| \mathbf{v}(\cdot, \mathbf{x})\right|_{G}<\delta\right\} \mathscr{E}\left\{\left|\mathbf{y}\left(t, \mathbf{x}, t_{0}, \mathbf{v}_{0}\right)\right|_{G}\right\}<\varepsilon$ and $\lim _{t \rightarrow \infty} \mathscr{E}\left\{\left|\mathbf{y}\left(t, \mathbf{x}, t_{0}, \mathbf{v}_{0}\right)\right|_{G}\right\}=0$.

The trivial solution of system (1) is said to be uniformly asymptotically stable in mean as to partial variables $\mathbf{y}$ if, for $\forall \varepsilon>0, \forall t_{0}>0$, there is $\delta(\varepsilon)$ such that for $\forall \mathbf{v}_{0} \epsilon$ $S_{\delta}=\left\{\left.\mathbf{v}|| \mathbf{v}(\cdot, \mathbf{x})\right|_{G}<\delta\right\} \mathscr{E}\left\{\left|\mathbf{y}\left(t, \mathbf{x}, t_{0}, \mathbf{v}_{0}\right)\right|_{G}\right\}<\varepsilon$ and $\lim _{t \rightarrow \infty} \mathscr{E}\left\{\left|\mathbf{y}\left(t, \mathbf{x}, t_{0}, \mathbf{v}_{0}\right)\right|_{G}\right\}=0$.

Definition 2. If $\mu(\cdot) \in C[[0, r], \mathbb{R}]$ is a strictly increasing function and $\mu(0)=0$, function $\mu$ is said to be class $\mathscr{K}$ function. Denote $\mu \in \mathscr{K}$ concisely. If $\mu(\cdot) \in C\left[\mathbb{R}^{+}, \mathbb{R}^{+}\right]$and $\mu \in \mathscr{K}, \lim _{r \rightarrow+\infty} \mu(r)=+\infty$, then $\mu \in \mathscr{K} R$.

A continuous function $V(t, \xi)$ is said to be positivedefinite if $V(t, 0)=0$ and, for some $\mu \in \mathscr{K} \mathscr{R}, V(t, \xi) \geq \mu(|\xi|)$. Write $C^{1,2}\left(\mathbb{R}_{+} \times \mathbb{R}^{n} ; \mathbb{R}_{+}\right)$for the family of all nonnegative functions $V(t, \xi)$ on $\mathbb{R}_{+} \times \mathbb{R}^{n}$ that are continuously twice differentiable in $\xi$ and once in $t$. If $V(t, \xi) \in C^{1,2}\left(\mathbb{R}_{+} \times \mathbb{R}^{n} ; \mathbb{R}_{+}\right)$, then define an operator $\mathscr{L} V(t, \xi)$ from $\mathbb{R}_{+} \times \mathbb{R}^{n}$ to $\mathbb{R}$ with respect to (1) by

$$
\begin{aligned}
\mathscr{L} V(t, \xi)= & V_{t}(t, \xi)+V_{\xi}^{T}(t, \xi) f(t, \mathbf{x}, \xi) \\
& +\frac{1}{2} \operatorname{Trace}\left[g^{T}(t, \mathbf{x}, \xi) V_{\xi \xi}(x, t) g(t, \mathbf{x}, \xi)\right],
\end{aligned}
$$

where

$$
\begin{gathered}
V_{t}(t, \xi)=\frac{\partial V(t, \xi)}{\partial t} \\
V_{\xi}^{T}(t, \xi)=\left(\frac{\partial V(t, \xi)}{\partial \xi_{1}}, \ldots, \frac{\partial V(t, \xi)}{\partial \xi_{n}}\right) \\
V_{\xi \xi}(x, t)=\left(\frac{\partial^{2} V(t, \xi)}{\partial \xi_{i} \partial \xi_{j}}\right)_{n \times n} .
\end{gathered}
$$


Applying the Itô formula to $\int_{G} \mathbf{V}(t, \mathbf{v}(t, \mathbf{x})) \mathrm{d} \mathbf{x}$ along system (1) gives for $\forall t \geq t_{0}$

$$
\begin{aligned}
\left.\left(\mathrm{d} \int_{G} V(t, \mathbf{v}(t, \mathbf{x})) \mathrm{d} \mathbf{x}\right)\right|_{(1)} \\
=\int_{G}\left[\left(\mathscr{L} V(t, \mathbf{v}(t, \mathbf{x}))+V_{\mathbf{v}}^{T}(t, \mathbf{v}) \rho \Delta \mathbf{v}(t, \mathbf{x})\right) \mathrm{d} t\right. \\
\left.\quad+V_{\mathbf{v}}^{T}(t, \mathbf{v}) g(t, \mathbf{x}, \mathbf{v}(t, \mathbf{x})) \mathrm{d} W(t)\right] \mathrm{d} \mathbf{x} .
\end{aligned}
$$

The existence of function $V(t, \mathbf{v}) \in C^{1,2}$ and another condition in the classical Lyapunov theorem on the stability of (1) are needed [30]. For convenience, similarly, we give the following definitions.

Definition 3. $V \in C^{1,2}\left(\mathbb{R}_{+} \times \mathbb{R}^{n} ; \mathbb{R}_{+}\right)$is called a LyapunovA function for (1), if $\mathscr{L} \int_{G} V(t, \mathbf{v}) \mathrm{d} \mathbf{x} \leq 0$, and is called a Lyapunov-B function for (1), if $\mathscr{L} \int_{G} V(t, \mathbf{v}) \mathrm{d} \mathbf{x} \leq$ $-b \int_{G} V(t, \mathbf{v}) \mathrm{d} \mathbf{x}$, in which $b>0$.

The following basic concepts and theorems on graph theory can be found in $[11,41]$. A directed graph $\mathscr{G}=(\mathscr{V}, E)$ contains a set $\mathscr{V}=\{1,2, \ldots, n\}$ of vertices and a set $E$ of $\operatorname{arcs}(i, j)$ leading from initial vertex $i$ to terminal vertex $j$. A subgraph $\mathscr{H}$ of $\mathscr{G}$ is said to be spanning if $\mathscr{H}$ and $\mathscr{G}$ have the same vertex set. A digraph $\mathscr{G}$ is weighted if each arc $(j, i)$ is assigned to a positive weight $a_{i j}$. Here $a_{i j}>0$ if and only if there exists an arc from vertex $j$ to vertex $i$ in $\mathscr{G}$. The weight $W(\mathscr{G})$ of $\mathscr{G}$ is the product of the weights on all its arcs. A directed path $\mathscr{P}$ in $\mathscr{G}$ is a subgraph with distinct vertices $\left\{i_{1}, i_{2}, \ldots, i_{m}\right\}$ such that its set of arcs is $\left\{\left(i_{k}, i_{k+1}\right)\right.$ : $k=1,2, \ldots, m-1\}$. If $i_{m}=i_{1}$, we call $\mathscr{P}$ a directed cycle. A connected subgraph $\mathscr{T}$ is a tree if it contains no cycles. A tree $\mathscr{T}$ is rooted at vertex $i$, called the root, if $i$ is not a terminal vertex of any arcs, and each of the remaining vertices is a terminal vertex of exactly one arc. A digraph $\mathscr{G}$ is strongly connected if, for any pair of distinct vertices, there exists a directed path from one to the other. Given a weighted digraph $\mathscr{G}$ with $n$ vertices, define the weight matrix $A=\left(a_{i j}\right)_{n \times n}$ whose entry $a_{i j}$ equals the weight of $\operatorname{arc}(j, i)$ if it exists and 0 otherwise. Denote the directed graph with weight matrix $A$ by $(\mathscr{G}, A)$. A weighted digraph $(\mathscr{G}, A)$ is said to be balanced if $W(\mathscr{C})=W(-\mathscr{C})$ for all directed cycles $\mathscr{C}$. Here, $-\mathscr{C}$ denotes the reverse of $\mathscr{C}$ and is constructed by reversing the direction of all arcs in $\mathscr{C}$. For a unicyclic graph $\mathbb{Q}$ with cycle $\mathscr{C}_{\mathscr{Q}}$, let $\widetilde{Q}$ be the unicyclic graph obtained by replacing $\mathscr{C}_{Q}$ with $-\mathscr{C}_{Q}$. Suppose that $(\mathscr{G}, A)$ is balanced; then $W(\mathscr{Q})=W(\widetilde{Q})$. The Laplacian matrix of $(\mathscr{G}, A)$ is defined as

$$
\mathscr{J}=\left(\begin{array}{cccc}
\sum_{i \neq 1} a_{1 i} & -a_{12} & \cdots & -a_{1 n} \\
-a_{21} & \sum_{i \neq 2} a_{2 i} & \cdots & -a_{2 n} \\
\vdots & \vdots & \ddots & \vdots \\
-a_{n 1} & -a_{n 2} & \cdots & \sum_{i \neq n} a_{i k}
\end{array}\right)
$$

Let $c_{i}$ denote the cofactor of the $i$ th diagonal element of $\mathscr{J}$.
Lemma 4 ([34] Kirchhoffs Matrix Tree Theorem). Assume $n \geq 2$. Then

$$
c_{i}=\sum_{\mathscr{T} \in \mathbb{\mathbb { V }}_{i}} W(\mathscr{T}), \quad i=1,2, \ldots, N,
$$

where $\mathbb{T}_{i}$ is the set of all spanning trees $\mathscr{T}$ of $(\mathscr{G}, A)$ that are rooted at vertex $i$. In particular, if $(\mathscr{G}, A)$ is strongly connected, then $c_{i}>0$ for $c_{i}=1,2, \ldots, n$.

Lemma 5 (see [11]). Assume $n \geq 2$. Let $c_{k}$ be given in (1). Then the following identity holds:

$$
\sum_{i, j=1}^{n} c_{i} a_{i j} F_{k j}\left(x_{i}, x_{j}\right)=\sum_{\mathbb{Q} \in \mathbb{Q}} W(Q) \sum_{(i, j) \in E\left(C_{Q}\right)} F_{i j}\left(x_{i}, x_{j}\right) .
$$

Here $F_{i j}\left(x_{i}, x_{j}\right), 1 \leq i, j \leq n$, are arbitrary functions, $\mathbb{Q}$ is the set of all spanning unicyclic graphs of $(\mathscr{G}, A), W(\mathbb{Q})$ is the weight of $Q$, and $C_{Q}$ denotes the directed cycle of $Q$.

\section{Main Results}

To begin with our main results, we will give an SCEDSN represented by digraph $\mathscr{G}$ with $N$ vertices, $N \geq 2$. In $i$ th vertex it is assigned a stochastic reaction-diffusion system

$$
\begin{gathered}
\mathrm{d} \mathbf{v}_{i}(t, \mathbf{x})=\left[\rho_{i}(t) \Delta \mathbf{v}_{i}(t, \mathbf{x})+f_{i}\left(t, \mathbf{x}, \mathbf{v}_{i}(t, \mathbf{x})\right)\right] \mathrm{d} t \\
+g_{i}\left(t, \mathbf{x}, \mathbf{v}_{i}(t, \mathbf{x})\right) \mathrm{d} W(t) \\
(t, \mathbf{x}) \in \mathbb{R}_{+} \times G
\end{gathered}
$$

where $\mathbf{v}_{i}(t, \mathbf{x})=\operatorname{col}\left(\mathbf{y}_{\mathbf{i}}, \mathbf{z}_{\mathbf{i}}\right) \in \mathbb{R}^{n_{i}}, \mathbf{y}_{\mathbf{i}} \in \mathbb{R}^{m_{i}}, \mathbf{z}_{\mathbf{i}} \in \mathbb{R}^{p_{i}}\left(m_{i}+p_{i}=\right.$ $\left.n_{i}\right), f_{i}: \mathbb{R}_{+} \times G \times \mathbb{R}^{n_{i}} \rightarrow \mathbb{R}^{n_{i}}$, and $g_{i}: \mathbb{R}_{+} \times G \times \mathbb{R}^{n_{i} \times m_{i}} \rightarrow$ $\mathbb{R}^{n_{i} \times m}$. If these systems are coupled, let

$$
\begin{array}{r}
H_{i j}: \mathbb{R}^{n_{i}} \times \mathbb{R}^{n_{j}} \times \mathbb{R} \longrightarrow \mathbb{R}^{n_{i}}, \\
N_{i j}: \mathbb{R}^{n_{i}} \times \mathbb{R}^{n_{j}} \times \mathbb{R} \longrightarrow \mathbb{R}^{n_{i} \times m}, \\
i, j=1,2, \ldots, N
\end{array}
$$

represent the influence of vertex $j$ on vertex $i$, and $H_{i j}=$ $N_{i j}=0$ if there exists no arc from $j$ to $i$ in $\mathscr{G}$. Then, by replacing $f_{i}$ and $g_{i}$ with $f_{i}+\sum_{j=1}^{N} H_{i j}$ and $g_{i}+\sum_{j=1}^{N} N_{i j}$, we get the following stochastic coupled system on graph $\mathscr{G}$ :

$$
\begin{aligned}
& \mathrm{d}_{i}(t, \mathbf{x}) \\
&= {\left[\rho_{i} \Delta \mathbf{v}_{i}(t, \mathbf{x})+f_{i}\left(t, \mathbf{x}, \mathbf{v}_{i}(t, \mathbf{x})\right)+\sum_{j=1}^{N} H_{i j}\left(\mathbf{v}_{i}, \mathbf{v}_{j}, t\right)\right] \mathrm{d} t } \\
&+\left[g_{i}\left(t, \mathbf{x}, \mathbf{v}_{i}(t, \mathbf{x})\right)+\sum_{j=1}^{N} N_{i j}\left(\mathbf{v}_{i}, \mathbf{v}_{j}, t\right)\right] \mathrm{d} W(t), \\
&(t, \mathbf{x}) \in \mathbb{R}_{+} \times G,
\end{aligned}
$$

$$
\frac{\partial \mathbf{v}_{i}(t, \mathbf{x})}{\partial \mathcal{N}}=0, \quad(t, \mathbf{x}) \in \mathbb{R}_{+} \times \partial G
$$


Without loss of generality, we suppose that functions $f_{i}$, $g_{i}, H_{i j}$, and $N_{i j}$ are such that initial-value problems to (10) and (12) have a unique solution and trivial solution $\mathbf{v}(t, \mathbf{x})=$ $\left(\mathbf{v}_{1}, \ldots, \mathbf{v}_{n}\right)=0$. Functions $f_{i}, f_{i}+\sum_{j=1}^{N} H_{i j}, g_{i}$ and $g_{i}+\sum_{j=1}^{N} N_{i j}$ meet Lipschitz condition with Lipschitz constant $L>0$. Functions $g_{i}$ and $g_{i}+\sum_{j=1}^{N} N_{i j}$ satisfy integral linear growth condition. Consider $\mathbf{y}=\sum_{i=1}^{N} \mathbf{y}_{\mathbf{i}}$. For $V_{i}\left(t, \mathbf{v}_{i}\right) \in C^{1,2}\left(\mathbb{R}_{+} \times\right.$ $\left.\mathbb{R}^{n_{i}} ; \mathbb{R}_{+}\right)$, define a differential operator $\mathscr{L} V_{i}\left(t, \mathbf{v}_{i}\right)$ associated with the $i$ th equation of (12) by

$$
\begin{aligned}
\mathscr{L} V_{i}\left(t, \mathbf{v}_{i}\right) \triangleq & \frac{\partial V_{i}\left(t, \mathbf{v}_{i}\right)}{\partial t}+\left(\frac{\partial V_{i}\left(t, \mathbf{v}_{i}\right)}{\partial \mathbf{v}_{i}}\right)^{T} \\
\times & {\left[f_{i}\left(t, \mathbf{x}, \mathbf{v}_{i}(t, \mathbf{x})\right)+\sum_{j=1}^{N} H_{i j}\left(\mathbf{v}_{i}, \mathbf{v}_{j}, t\right)\right] } \\
+\frac{1}{2} \operatorname{Trace}\{ & \left\{\left[g_{i}\left(t, \mathbf{x}, \mathbf{v}_{i}(t, \mathbf{x})\right)+\sum_{j=1}^{N} N_{i j}\left(\mathbf{v}_{i}, \mathbf{v}_{j}, t\right)\right]^{T}\right. \\
\times\left(V_{i}\left(t, \mathbf{v}_{i}\right)\right)_{\mathbf{v}_{i} \mathbf{v}_{j}}^{\prime \prime} & \\
\times & {\left.\left[g_{i}\left(t, \mathbf{x}, \mathbf{v}_{i}(t, \mathbf{x})\right)+\sum_{j=1}^{N} N_{i j}\left(\mathbf{v}_{i}, \mathbf{v}_{j}, t\right)\right]\right\} . }
\end{aligned}
$$

3.1. Stability in Mean. In this section, we will discuss stability in mean as to partial variables $\mathbf{y}$ of system (12) and draw some relevant conclusions.

Theorem 6. Let $\overline{\mathbf{v}}_{i}(t)=\int_{G} \mathbf{v}_{\mathbf{i}}(t, \mathbf{x}) d \mathbf{x}$. Suppose that the following conditions hold.

(A1) There exist positive-definite functions $V_{i}(t, \xi) \in$ $C^{1,2}\left(\mathbb{R}_{+} \times \mathbb{R}^{n_{i}} ; \mathbb{R}_{+}\right)$, functions $F_{i j}\left(\overline{\mathbf{v}}_{i}, \overline{\mathbf{v}}_{j}, t\right)$, and constants $a_{i j} \geq 0$ satisfying the following.

(I) $V_{i}\left(t, \overline{\mathbf{v}}_{i}(t)\right) \in C^{1,2}\left[\mathbb{R}_{+} \times S_{h}, \mathbb{R}\right],\left(S_{h}=\{\zeta|| \zeta(\cdot) \mid<\right.$ $h\})$ meeting $V_{i}(t, 0)=0$.

(II) $\mu_{1}\left(|\mathbf{y}(t, \mathbf{x})|_{G}\right) \leq V(t, \overline{\mathbf{v}}(t))$, where $V(t, \overline{\mathbf{v}}) \triangleq$ $\sum_{i=1}^{N} c_{i} V_{i}\left(t, \overline{\mathbf{v}}_{i}\right)$ and $c_{i}$ is defined as $(8) ; \mu_{1} \in \mathscr{K}$ is a convex function.

(III) $\mathscr{L} V_{i}\left(t, \overline{\mathbf{v}}_{i}\right) \leq \sum_{j=1}^{n} a_{i j} F_{i j}\left(\overline{\mathbf{v}}_{i}, \overline{\mathbf{v}}_{j}, t\right), t \geq 0, i=$ $1,2, \ldots, N$

(A2) Along each directed cycle $\mathbb{C}$ of the weighted digraph $(\mathbb{G}, A)$ in which $A=\left(a_{i j}\right)_{n \times n}$ there is

$$
\sum_{(i, j) \in E(\mathbb{C})} F_{i j}\left(\overline{\mathbf{v}}_{i}, \overline{\mathbf{v}}_{j}, t\right) \leq 0, \quad t \geq 0 .
$$

Then function $V(t, \overline{\mathbf{v}}) \triangleq \sum_{i=1}^{N} c_{i} V_{i}\left(t, \overline{\mathbf{v}}_{i}\right)$ is a Lyapunov- $A$ function for (12). Furthermore, the trivial solution of (12) is stable in mean as to partial variables $\mathbf{y}$.
Proof. It is not difficult to find that

$$
\begin{aligned}
& \mathrm{d}\left(\int_{G} \mathbf{v}_{\mathbf{i}}(t, \mathbf{x}) \mathrm{d} \mathbf{x}\right) \\
& =\int_{G}\left[\rho_{i}(t) \Delta \mathbf{v}_{i}(t, \mathbf{x})+f_{i}\left(t, \mathbf{x}, \mathbf{v}_{i}(t, \mathbf{x})\right)\right. \\
& \left.\quad+\sum_{j=1}^{N} H_{i j}\left(\mathbf{v}_{i}, \mathbf{v}_{j}, t\right)\right] \mathrm{d} \mathbf{x} \mathrm{d} t \\
& +\int_{G}\left[g_{i}\left(t, \mathbf{x}, \mathbf{v}_{i}(t, \mathbf{x})\right)+\sum_{j=1}^{N} N_{i j}\left(\mathbf{v}_{i}, \mathbf{v}_{j}, t\right)\right] \mathrm{d} \mathbf{x} \mathrm{d} W(t) .
\end{aligned}
$$

Applying Green formula, we deduce $\int_{G} \Delta \mathbf{v}_{\mathbf{i}}(t, \mathbf{x}) \mathrm{d} \mathbf{x}=$ $\int_{\partial G}\left(\partial \mathbf{v}_{\mathbf{i}} / \partial \mathcal{N}\right) \mathrm{d} s=0$. Hence, $(16)$ can be rewritten as

$$
\begin{aligned}
& \mathrm{d}\left(\int_{G} \mathbf{v}_{\mathbf{i}}(t, \mathbf{x}) \mathrm{d} \mathbf{x}\right) \\
& =\int_{G}\left[f_{i}\left(t, \mathbf{x}, \mathbf{v}_{i}(t, \mathbf{x})\right)+\sum_{j=1}^{N} H_{i j}\left(\mathbf{v}_{i}, \mathbf{v}_{j}, t\right)\right] \mathrm{d} \mathbf{x} \mathrm{d} t \\
& \quad+\int_{G}\left[g_{i}\left(t, \mathbf{x}, \mathbf{v}_{i}(t, \mathbf{x})\right)+\sum_{j=1}^{N} N_{i j}\left(\mathbf{v}_{i}, \mathbf{v}_{j}, t\right)\right] \mathrm{d} \mathbf{x} \mathrm{d} W(t) .
\end{aligned}
$$

Since $V_{i}\left(t_{0}, \overline{\mathbf{v}}_{i}\right)$ is continuous and $V_{i}\left(t_{0}, 0\right)=0$, we have $V(t, \mathbf{v}) \triangleq \sum_{i=1}^{N} c_{i} V_{i}\left(t, \mathbf{v}_{i}\right)$ that is continuous and $V(t, 0)=0$. Hence, there exists $\delta\left(t_{0}, \varepsilon\right)$ such that $V\left(t_{0}, \overline{\mathbf{v}}_{0}\right)<\mu_{1}(\varepsilon)$ when $\left|\overline{\mathbf{v}}_{0}\right|<\delta$. Choosing $\forall \mathbf{v}_{i 0}:\left|\overline{\mathbf{v}}_{i 0}\right|<\delta / N$ and applying Itô differential formula to $V_{i}\left(t, \overline{\mathbf{v}}_{i}\right)$ along the trajectory of system (17) yields

$$
\begin{aligned}
& \left.\mathrm{d} V_{i}\left(t, \overline{\mathbf{v}}_{i}\right)\right|_{(17)} \\
& =\frac{\partial V_{i}\left(t, \overline{\mathbf{v}}_{i}\right)}{\partial t} \mathrm{~d} t+\frac{\partial V\left(t, \overline{\mathbf{v}}_{i}\right)}{\partial \overline{\mathbf{v}}_{i}} d \overline{\mathbf{v}}_{i} \\
& +\frac{1}{2} \operatorname{Trace}\left[\int _ { G } \left[g_{i}\left(t, \mathbf{x}, \mathbf{v}_{i}(t, \mathbf{x})\right)\right.\right. \\
& \left.+\sum_{j=1}^{N} N_{i j}\left(\mathbf{v}_{i}, \mathbf{v}_{j}, t\right)\right]^{\top} \mathrm{d} \mathbf{x} \frac{\partial^{2} V\left(t, \overline{\mathbf{v}}_{i}\right)}{\partial \overline{\mathbf{v}}_{i} \partial \overline{\mathbf{v}}_{i}} \\
& \times \int_{G}\left[g_{i}\left(t, \mathbf{x}, \mathbf{v}_{i}(t, \mathbf{x})\right)\right.
\end{aligned}
$$




$$
\begin{gathered}
\left.\left.+\sum_{j=1}^{N} N_{i j}\left(\mathbf{v}_{i}, \mathbf{v}_{j}, t\right)\right] \mathrm{d} \mathbf{x}\right] \mathrm{d} t \\
=\mathscr{L} V\left(t, \overline{\mathbf{v}}_{i}\right) \mathrm{d} t+\frac{\partial V\left(t, \overline{\mathbf{v}}_{i}\right)}{\partial \overline{\mathbf{v}}_{i}} \\
\times \int_{G}\left[g_{i}\left(t, \mathbf{x}, \mathbf{v}_{i}(t, \mathbf{x})\right)+\sum_{j=1}^{N} N_{i j}\left(\mathbf{v}_{i}, \mathbf{v}_{j}, t\right)\right] \mathrm{d} \mathbf{x} \mathrm{d} W(t),
\end{gathered}
$$

where

$$
\begin{aligned}
& \mathscr{L} V_{i}\left(t, \overline{\mathbf{v}}_{i}\right) \triangleq \frac{\partial V_{i}\left(t, \overline{\mathbf{v}}_{i}\right)}{\partial t}+\left(\frac{\partial V_{i}\left(t, \overline{\mathbf{v}}_{i}\right)}{\partial \mathbf{v}_{i}}\right)^{T} \\
& \times\left[f_{i}\left(t, \mathbf{x}, \mathbf{v}_{i}(t, \mathbf{x})\right)+\sum_{j=1}^{N} H_{i j}\left(\mathbf{v}_{i}, \mathbf{v}_{j}, t\right)\right] \\
&+\frac{1}{2} \operatorname{Trace}\{[ {\left[g_{i}\left(t, \mathbf{x}, \mathbf{v}_{i}(t, \mathbf{x})\right)+\sum_{j=1}^{N} N_{i j}\left(\mathbf{v}_{i}, \mathbf{v}_{j}, t\right)\right]^{T} } \\
& \times\left(V_{i}\left(t, \mathbf{v}_{i}\right)\right)_{\mathbf{v}_{i} \mathbf{v}_{j}}^{\prime \prime} \\
&\left.\times\left[g_{i}\left(t, \mathbf{x}, \mathbf{v}_{i}(t, \mathbf{x})\right)+\sum_{j=1}^{N} N_{i j}\left(\mathbf{v}_{i}, \mathbf{v}_{j}, t\right)\right]\right\}
\end{aligned}
$$

for $\forall t \geq 0$. Hence,

$$
\begin{aligned}
& V_{i}\left(t, \overline{\mathbf{v}}_{i}\left(t, t_{0}, \overline{\mathbf{v}_{\mathbf{i} \mathbf{0}}}\right)\right) \\
& =V_{i}\left(t_{0}, \overline{\mathbf{v}_{\mathbf{i} \mathbf{0}}}\right)+\int_{t_{0}}^{t} \mathscr{L} V_{i}\left(s, \overline{\mathbf{v}}_{i}\left(s, t_{0}, \overline{\mathbf{v}_{\mathbf{i} \mathbf{0}}}\right)\right) \mathrm{d} s \\
& +\int_{t_{0}}^{t} \frac{\partial V_{i}\left(s, \overline{\mathbf{v}}_{i}\right)}{\partial \overline{\mathbf{v}}_{i}} \\
& \times \int_{G}\left[g_{i}\left(t, \mathbf{x}, \mathbf{v}_{i}(t, \mathbf{x})\right)\right. \\
& \left.+\sum_{j=1}^{N} N_{i j}\left(\mathbf{v}_{i}, \mathbf{v}_{j}, t\right)\right] \mathrm{d} \mathbf{x} \mathrm{d} w(s) .
\end{aligned}
$$

As $\partial V_{i}\left(t, \overline{\mathbf{v}}_{i}\right) / \partial \overline{\mathbf{v}}_{i}$ is continuous on $R_{+} \times S_{h}$ and $g_{i}(t, \mathbf{x}$, $\left.\mathbf{v}_{i}(t, \mathbf{x})\right)+\sum_{j=1}^{N} N_{i j}\left(\mathbf{v}_{i}, \mathbf{v}_{j}, t\right)$ satisfies integral linear growth condition, there must exist constant $L_{1}>0$ such that $\left|\partial V_{i}\left(t, \overline{\mathbf{v}}_{i}\right) / \partial \overline{\mathbf{v}}_{i}\right| \leq L_{1}$, so

$$
\begin{aligned}
& \left|\frac{\partial V_{i}\left(s, \overline{\mathbf{v}}_{i}\right)}{\partial \overline{\mathbf{v}}_{i}} \int_{G}\left[g_{i}\left(t, \mathbf{x}, \mathbf{v}_{i}(t, \mathbf{x})\right)+\sum_{j=1}^{N} N_{i j}\left(\mathbf{v}_{i}, \mathbf{v}_{j}, t\right)\right] \mathrm{d} \mathbf{x}\right| \\
& \quad \leq L_{1} K\left(1+\left|\int_{G} \mathbf{v}\left(t, \mathbf{x}, t_{0}, \mathbf{v}_{i 0}\right) \mathrm{d} \mathbf{x}\right|\right) \\
& \quad \leq L_{1} K(1+h) .
\end{aligned}
$$

By Theorem 2.8 of $[42,43]$, we obtain

$$
\begin{aligned}
\mathscr{E}\left[\int_{t_{0}}^{t} \frac{\partial V_{i}\left(s, \overline{\mathbf{v}}_{i}\right)}{\partial \overline{\mathbf{v}}_{i}} \int_{G}[\right. & g_{i}\left(t, \mathbf{x}, \mathbf{v}_{i}(t, \mathbf{x})\right) \\
& \left.\left.+\sum_{j=1}^{N} N_{i j}\left(\mathbf{v}_{i}, \mathbf{v}_{j}, t\right)\right] \mathrm{d} \mathbf{x} \mathrm{d} w(s)\right]=0 .
\end{aligned}
$$

On the other hand, by (A1)(III), it is derived that

$$
\mathscr{L} V(t, \overline{\mathbf{v}})=\sum_{i=1}^{N} c_{i} \mathscr{L} V_{i}\left(t, \overline{\mathbf{v}}_{i}\right) \leq \sum_{i, j=1} c_{i} a_{i j} F_{i j}\left(\overline{\mathbf{v}}_{i}, \overline{\mathbf{v}}_{j}, t\right) .
$$

Making use of Lemma 5 with weighted digraph $(\mathscr{S}, A)$, it yields

$$
\sum_{i, j=1} c_{i} a_{i j} F_{i j}\left(\overline{\mathbf{v}}_{i}, \overline{\mathbf{v}}_{j}, t\right)=\sum_{Q \in Q} W(\mathcal{Q}) \sum_{(i, j) \in E\left(C_{Q}\right)} F_{i j}\left(\overline{\mathbf{v}}_{i}, \overline{\mathbf{v}}_{j}, t\right) .
$$

In view of condition (A2) and a fact $W(\mathbb{Q})>0$, we get

$$
\mathscr{L} V(t, \overline{\mathbf{v}}) \leq \sum_{\mathbb{Q} \in \mathbb{Q}} W(\mathscr{Q}) \sum_{(i, j) \in E\left(C_{\mathscr{Q}}\right)} F_{i j}\left(\overline{\mathbf{v}}_{i}, \overline{\mathbf{v}}_{j}, t\right) \leq 0 .
$$

Thus $V(t, \overline{\mathbf{v}})$ is a Lyapunov-A function for (12). Taking the mathematical expectation at the two sides of (20) and using (22), (23), and (25) we have

$$
\mathscr{E}\left\{V\left(t, \mathbf{x}, \overline{\mathbf{v}}\left(t, t_{0}, \overline{\mathbf{v}_{0}}\right)\right)\right\} \leq V\left(t_{0}, \overline{\mathbf{v}_{0}}\right), \quad t \geq 0 .
$$

Combine Jensen inequality and condition (A1)(II),

$$
\begin{aligned}
\mu_{1}\left(\mathscr{E}\left(\left|\mathbf{y}\left(t, \mathbf{x}, t_{0}, \mathbf{v}_{0}\right)\right|_{G}\right)\right) & \leq \mathscr{E}\left(\mu_{1}\left(\left|\mathbf{y}\left(t, \mathbf{x}, t_{0}, \mathbf{v}_{0}\right)\right|_{G}\right)\right) \\
& \leq \mathscr{E}\left\{V\left(t, \overline{\mathbf{v}}\left(t, t_{0}, \overline{\mathbf{v}}_{0}\right)\right)\right\} \\
& \leq V\left(t_{0}, \overline{\mathbf{v}}_{0}\right) \leq \mu_{1}(\varepsilon) .
\end{aligned}
$$

Therefore $\mathscr{E}\left\{\left|\mathbf{y}\left(t, \mathbf{x}, t_{0}, \mathbf{v}_{0}\right)\right|_{G}\right\}<\varepsilon$. The proof is complete.

Note that if $(\mathscr{G}, A)$ is balanced, then

$$
\begin{aligned}
& \sum_{i, j=1} c_{i} a_{i j} F_{i j}\left(\overline{\mathbf{v}}_{i}, \overline{\mathbf{v}}_{j}, t\right) \\
& =\frac{1}{2} \sum_{\mathbb{Q} \in \mathbb{Q}} W(\mathscr{Q}) \sum_{(i, j) \in E\left(C_{\mathscr{Q}}\right)}\left[F_{i j}\left(\overline{\mathbf{v}}_{j}, \overline{\mathbf{v}}_{i}, t\right)+F_{j i}\left(\overline{\mathbf{v}}_{i}, \overline{\mathbf{v}}_{j}, t\right)\right] .
\end{aligned}
$$


In this case, condition (A2) is replaced by the following:

$$
\sum_{(i, j) \in E\left(C_{Q}\right)}\left[F_{i j}\left(\overline{\mathbf{v}}_{j}, \overline{\mathbf{v}}_{i}, t\right)+F_{j i}\left(\overline{\mathbf{v}}_{i}, \overline{\mathbf{v}}_{j}, t\right)\right] \leq 0 .
$$

Consequently, we get the following corollary.

Corollary 7. Suppose that $(\mathscr{G}, A)$ is balanced. Then the conclusion of Theorem 6 holds if (15) is replaced by (29).

Remark 8. Partial stability technique (stability of part of the variables) is most useful when a fully stabilized system losses some control engines or some phase variables are not actively controlled. However, the CSRDSNs are too complicated to derive the analytical solution. Therefore, it is of importance to work on the qualitative analysis of the system and how to construct an appropriate Lyapunov function is a key step. The proof shows that, if each vertex system of (12) has a globally stable trivial solution and possesses a Lyapunov function $V_{i}$, then the Lyapunov function for (12) can be systematically constructed by using individual $V_{i}$. Our results are new and extend some findings in [38], because our stability principle has a close relation to the topology property of the network.

Theorem 9. Assume that condition (A1) of Theorem 6 is substituted by the following.

(A3) There exist positive-definite functions $V_{i}(t, \xi) \quad \epsilon$ $C^{1,2}\left(\mathbb{R}_{+} \times \mathbb{R}^{n_{i}} ; \mathbb{R}_{+}\right)$, functions $F_{i j}\left(\overline{\mathbf{v}}_{i}, \overline{\mathbf{v}}_{j}, t\right)$, and constants $a_{i j} \geq 0$ satisfying the following.

(I) $V_{i}\left(t, \overline{\mathbf{v}}_{i}(t)\right) \in C^{1,2}\left[\mathbb{R}_{+} \times S_{h}, \mathbb{R}\right],\left(S_{h}=\{\zeta \mid\right.$ $|\zeta(\cdot)|<h\})$. (Here, function $V_{i}\left(t, \overline{\mathbf{v}}_{i}(t)\right)$ gets rid of the restriction of $\left.V_{i}(t, 0)=0\right)$.

(II) $\mu_{2}\left(|\mathbf{y}(t, \mathbf{x})|_{G}\right) \leq V(t, \overline{\mathbf{v}}(t)) \leq \mu_{3}\left(|\mathbf{v}(t, \mathbf{x})|_{G}\right)$, where $V(t, \overline{\mathbf{v}}) \triangleq \sum_{i=1}^{N} c_{i} V_{i}\left(t, \overline{\mathbf{v}}_{i}\right), c_{i}$ is defined as (8), $\mu_{2}, \mu_{3} \in \mathscr{K}$, and $\mu_{2}$ is a convex function.

(III) $\mathscr{L} V_{i}\left(t, \overline{\mathbf{v}}_{i}\right) \leq \sum_{j=1}^{n} a_{i j} F_{i j}\left(\overline{\mathbf{v}}_{i}, \overline{\mathbf{v}}_{j}, t\right), t \geq 0, i=$ $1,2, \ldots, N$.

Other conditions remain the same. Then function $V(t, \overline{\mathbf{v}}) \triangleq$ $\sum_{i=1}^{N} c_{i} V_{i}\left(t, \overline{\mathbf{v}}_{i}\right)$ is a Lyapunov-A function for (12). Furthermore, the trivial solution of (12) is uniformly stable in mean as to partial variables $\mathbf{y}$.

Proof. Since $V_{i}\left(t, \overline{\mathbf{v}}_{i}\right)$ is continuous, we have $V(t, \mathbf{v}) \triangleq$ $\sum_{i=1}^{N} c_{i} V_{i}\left(t, \mathbf{v}_{i}\right)$ that is continuous. Similar to the proof of Theorem 6 , we can obtain that $V(t, \overline{\mathbf{v}})$ is a Lyapunov-A function for (12) and

$$
\mathscr{E}\left\{V\left(t, \overline{\mathbf{v}}\left(t, t_{0}, \overline{\mathbf{v}}_{0}\right)\right)\right\} \leq V\left(t_{0}, \overline{\mathbf{v}}_{0}\right), \quad t \geq 0 .
$$

Let $\delta(\varepsilon)=\mu_{3}^{-1} \mu_{2}(\varepsilon)$. It follows from Jensen inequality and condition (A3)(II) that, for $\mathbf{v}_{0} \in S_{\delta}=\left\{\left.\mathbf{v}|| \mathbf{v}(\cdot, \mathbf{x})\right|_{G}<\delta(\varepsilon)\right\}$, we have

$$
\begin{aligned}
\mu_{2}(\mathscr{E} & \left.\left\{\left|\mathbf{y}\left(t, \mathbf{x}, t_{0}, \mathbf{v}_{0}\right)\right|_{G}\right\}\right) \\
& \leq \mathscr{E}\left\{V\left(t, \overline{\mathbf{v}}\left(t, t_{0}, \overline{\mathbf{v}}_{0}\right)\right)\right\} \leq V\left(t_{0}, \overline{\mathbf{v}}_{0}\right) \\
& \leq \mu_{3}\left(\left|\mathbf{v}_{0}(\cdot, \mathbf{x})\right|_{G}\right)<\mu_{2}(\varepsilon) .
\end{aligned}
$$

Therefore, we derive $E\left\{\left|\mathbf{y}\left(t, \mathbf{x}, t_{0}, \mathbf{v}_{0}\right)\right|_{G}\right\}<\varepsilon$ as required.
Corollary 10. Suppose that $(\mathscr{G}, A)$ is balanced. Then the conclusion of Theorem 9 holds if (15) is replaced by (29).

3.2. Asymptotical Stability in Mean. In this section, some sufficient principles are established for asymptotic stability in mean and uniformly asymptotic stability in mean as to partial variables.

Theorem 11. Let $\overline{\mathbf{v}}_{i}(t)=\int_{G} \mathbf{v}_{\mathbf{i}}(t, \mathbf{x}) d \mathbf{x}$. Suppose that the following conditions hold.

(B1) There exist positive-definite functions $V_{i}(t, \xi) \quad \epsilon$ $C^{1,2}\left(\mathbb{R}_{+} \times \mathbb{R}^{n_{i}} ; \mathbb{R}_{+}\right)$, functions $F_{i j}\left(\overline{\mathbf{v}}_{i}, \overline{\mathbf{v}}_{j}, t\right)$, and constants $a_{i j} \geq 0$ satisfying the following.

(I) $V_{i}\left(t, \overline{\mathbf{v}}_{i}(t)\right) \in C^{1,2}\left[\mathbb{R}_{+} \times S_{h}, \mathbb{R}\right],\left(S_{h}=\{\zeta|| \zeta(\cdot) \mid<\right.$ $h\})$ meeting $V_{i}(t, 0)=0$.

(II) $\mu_{4}\left(|\mathbf{y}(t, \mathbf{x})|_{G}\right) \leq V(t, \overline{\mathbf{v}}(t))$, where $V(t, \overline{\mathbf{v}}) \triangleq$ $\sum_{i=1}^{N} c_{i} V_{i}\left(t, \overline{\mathbf{v}}_{i}\right)$ and $c_{i}$ is defined as $(8) ; \mu_{4} \in \mathscr{K}$ is a convex function.

(III) $\mathscr{L} V_{i}\left(t, \overline{\mathbf{v}}_{i}\right) \leq-b_{i} V_{i}\left(t, \overline{\mathbf{v}}_{i}\right)+\sum_{j=1}^{n} a_{i j} F_{i j}\left(\overline{\mathbf{v}}_{i}, \overline{\mathbf{v}}_{j}, t\right)$, constants $b_{i} \geq 0, t \geq 0, i=1,2, \ldots, N$.

(B2) Condition (A2) holds, if $(\mathscr{G}, A)$ is balanced and (29) holds.

Then, function $V(t, \overline{\mathbf{v}}) \triangleq \sum_{i=1}^{N} c_{i} V_{i}\left(t, \overline{\mathbf{v}}_{i}\right)$ is a Lyapunov- $B$ function for (12). Consequently, the trivial solution of (12) is asymptotically stable in mean as to partial variables $\mathbf{y}$.

Proof. We can show in the same way as in the proof of Theorem 6 that

$$
\mathscr{L} V(t, \overline{\mathbf{v}})=\sum_{i=1}^{N} c_{i} \mathscr{L} V_{i}\left(t, \overline{\mathbf{v}}_{i}\right) \leq-b V(t, \overline{\mathbf{v}}),
$$

where $b=\min \left\{b_{1}, b_{2}, \ldots, b_{N}\right\}$. Hence, we conclude that function $V(t, \overline{\mathbf{v}})$ is a Lyapunov-B function for (12). From Theorem 6, it is easy to derive that the trivial solution of system (12) is stable in mean as to partial variables y. So the following task is to prove

$$
\lim _{t \rightarrow \infty} \mathscr{E}\left\{\left|\mathbf{y}\left(t, \mathbf{x}, t_{0}, \mathbf{v}_{0}\right)\right|_{G}\right\}=0
$$

only. Similar to the proof of Theorem 6, it is not difficult to derive

$$
\begin{aligned}
\mathscr{E} V_{i}(t & \left., \overline{\mathbf{v}}_{i}\left(t, t_{0}, \overline{\mathbf{v}}_{i 0}\right)\right) \\
& =V_{i}\left(t_{0}, \overline{\mathbf{v}}_{i 0}\right)+\int_{t_{0}}^{t} \mathscr{E} \mathscr{L} V_{i}\left(s, \overline{\mathbf{v}}_{i}\left(s, t_{0}, \overline{\mathbf{v}}_{i 0}\right)\right) \mathrm{d} s .
\end{aligned}
$$

Then, we obtain that $V(t, \overline{\mathbf{v}})$ is a Lyapunov-A function for (12) and

$$
\mathscr{E}\left\{V\left(t, \overline{\mathbf{v}}\left(t, t_{0}, \overline{\mathbf{v}}_{0}\right)\right)\right\} \leq V\left(t_{0}, \overline{\mathbf{v}}_{0}\right), \quad t \geq 0 .
$$

Here we need to reduce to absurdity. Suppose

$$
\lim _{t \rightarrow \infty} \mathscr{E}\left\{\left|\mathbf{y}\left(t, \mathbf{x}, t_{0}, \mathbf{v}_{0}\right)\right|_{G}\right\} \neq 0 .
$$


Instead

$$
\lim _{t \rightarrow \infty} \mathscr{E}\left\{\left|\mathbf{y}\left(t, \mathbf{x}, t_{0}, \mathbf{v}_{0}\right)\right|_{G}\right\} \triangleq \widehat{\lambda}_{\infty}>0
$$

Combining condition (B1)(II) of Theorem 11, we obtain

$$
\begin{aligned}
\mu_{4}(\mathscr{E} & \left.\left\{\left|\mathbf{y}\left(t, \mathbf{x}, t_{0}, \mathbf{v}_{0}\right)\right|_{G}\right\}\right) \\
& \leq \mathscr{E}\left\{V\left(t, \overline{\mathbf{v}}\left(t, t_{0}, \overline{\mathbf{v}}_{0}\right)\right)\right\} \\
& \leq V\left(t_{0}, \overline{\mathbf{v}}_{0}\right)-\int_{t_{0}}^{t} \mu_{4}\left(\mathscr{E}\left\{\left|\mathbf{y}\left(s, \mathbf{x}, t_{0}, \mathbf{v}_{0}\right)\right|_{G}\right\}\right) \mathrm{d} s .
\end{aligned}
$$

Therefore,

$$
\begin{aligned}
0 & <\lim _{t \rightarrow \infty} \alpha\left(\mathscr{E}\left\{\left|\mathbf{y}\left(t, \mathbf{x}, t_{0}, \mathbf{v}_{0}\right)\right|_{G}\right\}\right) \\
& \leq V\left(t_{0}, \overline{\mathbf{v}}_{0}\right)-\int_{t_{0}}^{t} \lim _{t \rightarrow \infty} \mu_{4}\left(\mathscr{E}\left\{\left|\mathbf{y}\left(s, \mathbf{x}, t_{0}, \mathbf{v}_{0}\right)\right|_{G}\right\}\right) \mathrm{d} s \\
& =V\left(t_{0}, \overline{\mathbf{v}}_{0}\right)-\mu_{4}\left(\hat{\lambda}_{\infty}\right)\left(t-t_{0}\right) .
\end{aligned}
$$

However, it is obvious that (39) can not be satisfied as $t \gg$ $t_{0}$. Thus, hypothesis $\lim _{t \rightarrow \infty} \mathscr{E}\left\{\left|\mathbf{y}\left(t, \mathbf{x}, t_{0}, \mathbf{v}_{0}\right)\right|_{G}\right\} \neq 0$ does not come into existence. It should be

$$
\lim _{t \rightarrow \infty} \mathscr{E}\left\{\left|\mathbf{y}\left(t, \mathbf{x}, t_{0}, \mathbf{v}_{0}\right)\right|_{G}\right\}=0
$$

as required; that is, the trivial solution of system (12) is asymptotically stable in mean as to partial variables $\mathbf{y}$. This completes the proof.

Theorem 12. Let $\overline{\mathbf{v}}_{i}(t)=\int_{G} \mathbf{v}_{\mathbf{i}}(t, \mathbf{x}) d \mathbf{x}$. Suppose that the following conditions hold.

(B3) There exist positive-definite functions $V_{i}(t, \xi) \in$ $C^{1,2}\left(\mathbb{R}_{+} \times \mathbb{R}^{n_{i}} ; \mathbb{R}_{+}\right)$, functions $F_{i j}\left(\overline{\mathbf{v}}_{i}, \overline{\mathbf{v}}_{j}, t\right)$, and constants $a_{i j} \geq 0$ satisfying the following.

(I) $V_{i}\left(t, \overline{\mathbf{v}}_{i}(t)\right) \in C^{1,2}\left[\mathbb{R}_{+} \times S_{h}, \mathbb{R}\right],\left(S_{h}=\{\zeta|| \zeta(\cdot) \mid<\right.$ $h$ ) meeting $V_{i}(t, 0)=0$.

(II) $\mu_{4}\left(|\mathbf{y}(t, \mathbf{x})|_{G}\right) \leq V(t, \overline{\mathbf{v}}(t))$, where $V(t, \overline{\mathbf{v}}) \triangleq$ $\sum_{i=1}^{N} c_{i} V_{i}\left(t, \overline{\mathbf{v}}_{i}\right)$ and $c_{i}$ is defined as $(8) ; \mu_{4} \in \mathscr{K}$ is a convex function.
(III) $\mathscr{L} V_{i}\left(t, \overline{\mathbf{v}}_{i}\right) \quad \leq \quad-\mu_{5}\left(|\mathbf{y}(t, \mathbf{x})|_{G}\right) \quad+$ $\sum_{j=1}^{n} a_{i j} F_{i j}\left(\overline{\mathbf{v}}_{i}, \overline{\mathbf{v}}_{j}, t\right), \mu_{5} \in \mathscr{K}$ is a convex function, $t \geq 0, i=1,2, \ldots, N$.

(B2) Condition (A2) holds, if $(\mathscr{G}, A)$ is balanced and (29) holds.

Then, function $V(t, \overline{\mathbf{v}}) \triangleq \sum_{i=1}^{N} c_{i} V_{i}\left(t, \overline{\mathbf{v}}_{i}\right)$ is a Lyapunov- $A$ function for (12). Consequently, the trivial solution of (12) is asymptotically stable in mean as to partial variables $\mathbf{y}$.

Remark 13. Similar to the proof of Theorems 6 and 11, we can easily proof Theorem 12 . Please note that, in Theorem 11, we can construct a Lyapunov-B function for (12), but in Theorem 12 only a Lyapunov-A function for (12). Further, note the fact that $-\mu_{5}\left(|\mathbf{v}(t, \mathbf{x})|_{G}\right) \leq-\mu_{5}\left(|\mathbf{y}(t, \mathbf{x})|_{G}\right)$. We can draw the following theorem immediately.
Corollary 14. Suppose that in Theorem 12, condition (B3)(III) is replaced by

$$
\begin{gathered}
(I I I)^{\prime} \quad \mathscr{L} V_{i}\left(t, \overline{\mathbf{v}}_{i}\right) \leq-\mu_{5}\left(|\mathbf{v}(t, \mathbf{x})|_{G}\right)+\sum_{j=1}^{n} a_{i j} F_{i j}\left(\overline{\mathbf{v}}_{i}, \overline{\mathbf{v}}_{j}, t\right), \\
\mu_{5} \in \mathscr{K} \text { is a convex function, } t \geq 0, i=1,2, \ldots, N
\end{gathered}
$$

Other conditions remain the same. Then the conclusion of Theorem 12 holds.

The foregoing are all concerned with asymptotic stability as to partial variables. The following is focused on uniformly asymptotic stability as to partial variables.

Theorem 15. Assume that condition (B3) of Theorem 12 is substituted by the following.

(B4) There exist positive-definite functions $V_{i}(t, \xi) \in$ $C^{1,2}\left(\mathbb{R}_{+} \times \mathbb{R}^{n_{i}} ; \mathbb{R}_{+}\right)$, functions $F_{i j}\left(\overline{\mathbf{v}}_{i}, \overline{\mathbf{v}}_{j}, t\right)$, and constants $a_{i j} \geq 0$ satisfying the following.

(I) $V_{i}\left(t, \overline{\mathbf{v}}_{i}(t)\right) \in C^{1,2}\left[\mathbb{R}_{+} \times S_{h}, \mathbb{R}\right],\left(S_{h}=\{\zeta \mid\right.$ $|\zeta(\cdot)|<h\}$ ). (Here, function $V_{i}\left(t, \overline{\mathbf{v}}_{i}(t)\right)$ gets rid of the restriction of $\left.V_{i}(t, 0)=0\right)$.

(II) $\mu_{6}\left(|\mathbf{y}(t, \mathbf{x})|_{G}\right) \leq V(t, \overline{\mathbf{v}}(t)) \leq \mu_{7}\left(|\mathbf{v}(t, \mathbf{x})|_{G}\right)$, where $V(t, \overline{\mathbf{v}}) \triangleq \sum_{i=1}^{N} c_{i} V_{i}\left(t, \overline{\mathbf{v}}_{i}\right), c_{i}$ is defined as $(8), \mu_{7} \in$ $\mathscr{K}$, and $\mu_{6} \in \mathscr{K}$ is a convex function.
(III) $\mathscr{L} V_{i}\left(t, \overline{\mathbf{v}}_{i}\right) \quad \leq \quad-\mu_{8}\left(|\mathbf{y}(t, \mathbf{x})|_{G}\right) \quad+$ $\sum_{j=1}^{n} a_{i j} F_{i j}\left(\overline{\mathbf{v}}_{i}, \overline{\mathbf{v}}_{j}, t\right), \mu_{8} \in \mathscr{K}$ is a convex function, $t \geq 0, i=1,2, \ldots, N$.

Other conditions remain the same. Then function $V(t, \overline{\mathbf{v}}) \triangleq$ $\sum_{i=1}^{N} c_{i} V_{i}\left(t, \overline{\mathbf{v}}_{i}\right)$ is a Lyapunov-A function for (12). Furthermore, the trivial solution of (12) is uniformly asymptotically stable in mean as to partial variables $\mathbf{y}$.

Proof. Because the conditions of Theorem 15 cover those of Theorem 9, it is obvious that the trivial solution of system (1) is uniformly stable as to partial variables $\mathbf{y}$. Now, we only need to prove

$$
\lim _{t \rightarrow \infty} \mathscr{E}\left\{\left|\mathbf{y}\left(t, \mathbf{x}, t_{0}, \mathbf{v}_{0}\right)\right|_{G}\right\}=0 .
$$

Similar to the proof of Theorem 11, here we need to reduce to absurdity. Suppose

$$
\lim _{t \rightarrow \infty} \mathscr{E}\left\{\left|\mathbf{y}\left(t, \mathbf{x}, t_{0}, \mathbf{v}_{0}\right)\right|_{G}\right\} \neq 0 .
$$

Instead

$$
\lim _{t \rightarrow \infty} \mathscr{E}\left\{\left|\mathbf{y}\left(t, \mathbf{x}, t_{0}, \mathbf{v}_{0}\right)\right|_{G}\right\} \triangleq \hat{\lambda}_{\infty}>0 .
$$

Combining condition (B1)(II) of Theorem 15, we obtain

$$
\begin{aligned}
\mu_{6}(\mathscr{E} & \left.\left\{\left|\mathbf{y}\left(t, \mathbf{x}, t_{0}, \mathbf{v}_{0}\right)\right|_{G}\right\}\right) \\
& \leq \mathscr{E}\left\{V\left(t, \overline{\mathbf{v}}\left(t, t_{0}, \overline{\mathbf{v}}_{0}\right)\right)\right\} \\
& \leq V\left(t_{0}, \overline{\mathbf{v}}_{0}\right)-\int_{t_{0}}^{t} \mu_{6}\left(\mathscr{E}\left\{\left|\mathbf{y}\left(s, \mathbf{x}, t_{0}, \mathbf{v}_{0}\right)\right|_{G}\right\}\right) \mathrm{d} s .
\end{aligned}
$$


Therefore,

$$
\begin{aligned}
0 & <\lim _{t \rightarrow \infty} \alpha\left(\mathscr{E}\left\{\left|\mathbf{y}\left(t, \mathbf{x}, t_{0}, \mathbf{v}_{0}\right)\right|_{G}\right\}\right) \\
& \leq V\left(t_{0}, \overline{\mathbf{v}}_{0}\right)-\int_{t_{0}}^{t} \lim _{t \rightarrow \infty} \mu_{6}\left(\mathscr{E}\left\{\left|\mathbf{y}\left(s, \mathbf{x}, t_{0}, \mathbf{v}_{0}\right)\right|_{G}\right\}\right) \mathrm{d} s \\
& =V\left(t_{0}, \overline{\mathbf{v}}_{0}\right)-\mu_{6}\left(\hat{\lambda}_{\infty}\right)\left(t-t_{0}\right) .
\end{aligned}
$$

However, it is obvious that (46) cannot be satisfied as $t \gg$ $t_{0}$. Thus, hypothesis $\lim _{t \rightarrow \infty} \mathscr{E}\left\{\left|\mathbf{y}\left(t, \mathbf{x}, t_{0}, \mathbf{v}_{0}\right)\right|_{G}\right\} \neq 0$ does not come into existence. It should be

$$
\lim _{t \rightarrow \infty} \mathscr{E}\left\{\left|\mathbf{y}\left(t, \mathbf{x}, t_{0}, \mathbf{v}_{0}\right)\right|_{G}\right\}=0
$$

as required; that is, the trivial solution of system (12) is uniformly asymptotically stable in mean as to partial variables $\mathbf{y}$. This completes the proof.

Note the fact that $-\mu_{8}\left(|\mathbf{v}(t, \mathbf{x})|_{G}\right) \leq-\mu_{8}\left(|\mathbf{y}(t, \mathbf{x})|_{G}\right)$. We can still derive another conclusion as follows.

Corollary 16. Suppose that, in Theorem 15, condition (B4)(III) is replaced by

$$
(I I I)^{\prime} \mathscr{L} V_{i}\left(t, \overline{\mathbf{v}}_{i}\right) \leq-\mu_{8}\left(|\mathbf{v}(t, \mathbf{x})|_{G}\right)+\sum_{j=1}^{n} a_{i j} F_{i j}\left(\overline{\mathbf{v}}_{i}, \overline{\mathbf{v}}_{j}, t\right),
$$$$
\mu_{8} \in \mathscr{K} \text { is a convex function, } t \geq 0, i=1,2, \ldots, N \text {. }
$$

Other conditions remain the same. Then the conclusion of Theorem 15 holds.

Theorem 17. Assume that condition (B2) of Theorem 11 is substituted by the following.

(B5) There exist positive-definite functions $V_{i}(t, \xi) \quad \epsilon$ $C^{1,2}\left(\mathbb{R}_{+} \times \mathbb{R}^{n_{i}} ; \mathbb{R}_{+}\right)$, functions $F_{i j}\left(\overline{\mathbf{v}}_{i}, \overline{\mathbf{v}}_{j}, t\right)$, and constants $a_{i j} \geq 0$ satisfying the following.

(I) $V_{i}\left(t, \overline{\mathbf{v}}_{i}(t)\right) \in C^{1,2}\left[\mathbb{R}_{+} \times S_{h}, \mathbb{R}\right],\left(S_{h}=\{\zeta \mid\right.$ $|\zeta(\cdot)|<h\}$ ). (Here, function $V_{i}\left(t, \overline{\mathbf{v}}_{i}(t)\right)$ gets rid of the restriction of $\left.V_{i}(t, 0)=0\right)$.

(II) $\mu_{9}\left(|\mathbf{y}(t, \mathbf{x})|_{G}\right) \leq V(t, \overline{\mathbf{v}}(t)) \leq \mu_{10}\left(|\mathbf{v}(t, \mathbf{x})|_{G}\right)$, where $V(t, \overline{\mathbf{v}}) \triangleq \sum_{i=1}^{N} c_{i} V_{i}\left(t, \overline{\mathbf{v}}_{i}\right), c_{i}$ is defined as (8), $\mu_{10} \in \mathscr{K}$, and $\mu_{9} \in \mathscr{K}$ is a convex function.

(III) $\mathscr{L} V_{i}\left(t, \overline{\mathbf{v}}_{i}\right) \leq-\bar{b}_{i} V_{i}\left(t, \overline{\mathbf{v}}_{i}\right)+\sum_{j=1}^{n} a_{i j} F_{i j}\left(\overline{\mathbf{v}}_{i}, \overline{\mathbf{v}}_{j}, t\right)$, constants $\bar{b}_{i} \geq 0, \quad t \geq 0, i=1,2, \ldots, N$.

Other conditions remain the same. Then function $V(t, \overline{\mathbf{v}}) \triangleq$ $\sum_{i=1}^{N} c_{i} V_{i}\left(t, \overline{\mathbf{v}}_{i}\right)$ is a Lyapunov-B function for (12). Furthermore, the trivial solution of (12) is uniformly asymptotically stable in mean as to partial variables $\mathbf{y}$.

3.3. Exponential Stability in Mean. In this section, we will discuss exponential stability in mean of the trivial solution of system (12) as to partial variables.
Theorem 18. Let $\overline{\mathbf{v}}_{i}(t)=\int_{G} \mathbf{v}_{i}(t, \mathbf{x}) d \mathbf{x}$. Suppose that the following conditions hold.

(C1) There exist positive-definite functions $V_{i}(t, \xi) \quad \epsilon$ $C^{1,2}\left(\mathbb{R}_{+} \times \mathbb{R}^{n_{i}} ; \mathbb{R}_{+}\right)$, functions $F_{i j}\left(\overline{\mathbf{v}}_{i}, \overline{\mathbf{v}}_{j}, t\right)$, and constants $a_{i j} \geq 0$ satisfying the following.

(I) $V_{i}\left(t, \overline{\mathbf{v}}_{i}(t)\right) \in C^{1,2}\left[\mathbb{R}_{+} \times S_{h}, \mathbb{R}\right],\left(S_{h}=\{\zeta \mid\right.$ $|\zeta(\cdot)|<h\}$ ). (Here, function $V_{i}\left(t, \overline{\mathbf{v}}_{i}(t)\right)$ gets rid of the restriction of $\left.V_{i}(t, 0)=0\right)$.

(II) $\left.\bar{c}_{1}|\mathbf{y}(t, \mathbf{x})|_{G}\right) \leq V(t, \overline{\mathbf{v}}(t)) \leq \bar{c}_{2}|\mathbf{v}(t, \mathbf{x})|_{G}$, where $V(t, \overline{\mathbf{v}}) \triangleq \sum_{i=1}^{N} c_{i} V_{i}\left(t, \overline{\mathbf{v}}_{i}\right), c_{i}$ is defined as (8), and $\bar{c}_{1}$ and $\bar{c}_{2}$ are positive constants.

(III) $\mathscr{L} V_{i}\left(t, \overline{\mathbf{v}}_{i}\right) \leq-\widehat{b}_{i} V_{i}\left(t, \overline{\mathbf{v}}_{i}\right)+\sum_{j=1}^{n} a_{i j} F_{i j}\left(\overline{\mathbf{v}}_{i}, \overline{\mathbf{v}}_{j}, t\right)$, constants $\widehat{b}_{i}>0, \quad t \geq 0, i=1,2, \ldots, N$.

(C2) Condition (A2) holds, if $(\mathscr{G}, A)$ is balanced and (29) holds.

Then, function $V(t, \overline{\mathbf{v}}) \triangleq \sum_{i=1}^{N} c_{i} V_{i}\left(t, \overline{\mathbf{v}}_{i}\right)$ is a Lyapunov- $B$ function for (12), and

$$
E\left\{|\mathbf{y}(t, \mathbf{x})|_{G}\right\} \leq \frac{\bar{c}_{2}}{\bar{c}_{1}}\left|v_{0}\right|_{G} e^{-\widehat{b}\left(t-t_{0}\right)}, \quad t \geq t_{0}
$$

where $\widehat{b}=\min \left\{\widehat{b}_{1}, \ldots, \widehat{b}_{N}\right\}$; that is, the trivial solution of system (12) is exponentially stable in mean as to partial variables $\mathbf{y}$.

Proof. We can show in the same way as in the proof of Theorem 6 that

$$
\mathscr{L} V(t, \overline{\mathbf{v}})=\sum_{i=1}^{N} c_{i} \mathscr{L} V_{i}\left(t, \overline{\mathbf{v}}_{i}\right) \leq-\widehat{b} V(t, \overline{\mathbf{v}})
$$

where $\widehat{b}=\min \left\{\widehat{b}_{1}, \widehat{b}_{2}, \ldots, \widehat{b}_{N}\right\}$. Hence, we conclude that function $V(t, \overline{\mathbf{v}})$ is a Lyapunov-B function for (12). Integrating system (12) as to spatial variables $\mathbf{x}$ gives

$$
\begin{aligned}
& \mathrm{d}\left(\int_{G} \mathbf{v}_{\mathbf{i}}(t, \mathbf{x}) \mathrm{d} \mathbf{x}\right) \\
& =\int_{G}\left[\rho_{i}(t) \Delta \mathbf{v}_{i}(t, \mathbf{x})+f_{i}\left(t, \mathbf{x}, \mathbf{v}_{i}(t, \mathbf{x})\right)\right. \\
& \left.\quad+\sum_{j=1}^{N} H_{i j}\left(\mathbf{v}_{i}, \mathbf{v}_{j}, t\right)\right] \mathrm{d} \mathbf{x} \mathrm{d} t \\
& +\int_{G}\left[g_{i}\left(t, \mathbf{x}, \mathbf{v}_{i}(t, \mathbf{x})\right)+\sum_{j=1}^{N} N_{i j}\left(\mathbf{v}_{i}, \mathbf{v}_{j}, t\right)\right] \mathrm{d} \mathbf{x} \mathrm{d} W(t) .
\end{aligned}
$$


Combining Green formula and boundary condition we deduce $\int_{G} \Delta \mathbf{v}_{\mathbf{i}}(t, \mathbf{x}) \mathrm{d} \mathbf{x}=\int_{\partial G}\left(\partial \mathbf{v}_{\mathbf{i}} / \partial \mathcal{N}\right) \mathrm{d} s=0$. Hence, (51) can be rewritten as

$$
\begin{aligned}
& \mathrm{d}\left(\int_{G} \mathbf{v}_{\mathbf{i}}(t, \mathbf{x}) \mathrm{d} \mathbf{x}\right) \\
& =\int_{G}\left[f_{i}\left(t, \mathbf{x}, \mathbf{v}_{i}(t, \mathbf{x})\right)+\sum_{j=1}^{N} H_{i j}\left(\mathbf{v}_{i}, \mathbf{v}_{j}, t\right)\right] \mathrm{d} \mathbf{x} \mathrm{d} t \\
& \quad+\int_{G}\left[g_{i}\left(t, \mathbf{x}, \mathbf{v}_{i}(t, \mathbf{x})\right)+\sum_{j=1}^{N} N_{i j}\left(\mathbf{v}_{i}, \mathbf{v}_{j}, t\right)\right] \mathrm{d} \mathbf{x} \mathrm{d} W(t) .
\end{aligned}
$$

Since $V_{i}\left(t_{0}, \overline{\mathbf{v}}_{i}\right)$ is continuous and $V_{i}\left(t_{0}, 0\right)=0$, we have $V(t, \overline{\mathbf{v}}) \triangleq \sum_{i=1}^{N} c_{i} V_{i}\left(t, \mathbf{v}_{i}\right)$ that is continuous and $V(t, 0)=0$. Hence, there exists $\delta\left(t_{0}, \varepsilon\right)$ such that $V\left(t_{0}, \overline{\mathbf{v}}_{0}\right)<\mu_{1}(\varepsilon)$ when $\left|\overline{\mathbf{v}}_{0}\right|<\delta$. Choosing $\forall \mathbf{v}_{i 0}:\left|\overline{\mathbf{v}}_{i 0}\right|<\delta / N$ and applying Itô differential formula to $V_{i}\left(t, \overline{\mathbf{v}}_{i}\right)$ along the trajectory of system (17) yields

$$
\begin{aligned}
& \left.\mathrm{d} V_{i}\left(t, \overline{\mathbf{v}}_{i}\right)\right|_{(17)} \\
& =\frac{\partial V_{i}\left(t, \overline{\mathbf{v}}_{i}\right)}{\partial t} \mathrm{~d} t+\frac{\partial V\left(t, \overline{\mathbf{v}}_{i}\right)}{\partial \bar{v}_{i}} \mathrm{~d} \overline{\mathbf{v}}_{i} \\
& +\frac{1}{2} \operatorname{Trace}\left[\int _ { G } \left[g_{i}\left(t, \mathbf{x}, \mathbf{v}_{i}(t, \mathbf{x})\right)\right.\right. \\
& \left.+\sum_{j=1}^{N} N_{i j}\left(\mathbf{v}_{i}, \mathbf{v}_{j}, t\right)\right]^{\top} \mathrm{d} \mathbf{x} \frac{\partial^{2} V\left(t, \overline{\mathbf{v}}_{i}\right)}{\partial \overline{\mathbf{v}}_{i} \partial \overline{\mathbf{v}}_{i}} \\
& \left.\left.+\sum_{j=1}^{N} N_{i j}\left(\mathbf{v}_{i}, \mathbf{v}_{j}, t\right)\right] \mathrm{d} \mathbf{x}\right] \mathrm{d} t \\
& =\mathscr{L} V\left(t, \overline{\mathbf{v}}_{i}\right) \mathrm{d} t+\frac{\partial V\left(t, \overline{\mathbf{v}}_{i}\right)}{\partial \overline{\mathbf{v}}_{i}} \\
& \quad \times \int_{G}\left[g_{i}\left(t, \mathbf{x}, \mathbf{v}_{i}(t, \mathbf{x})\right)+\sum_{j=1}^{N} N_{i j}\left(\mathbf{v}_{i}, \mathbf{v}_{j}, t\right)\right] \mathrm{d} \mathbf{x} \mathrm{d} W(t),
\end{aligned}
$$

where

$$
\begin{gathered}
\mathscr{L} V_{i}\left(t, \overline{\mathbf{v}}_{i}\right) \triangleq \frac{\partial V_{i}\left(t, \overline{\mathbf{v}}_{i}\right)}{\partial t}+\left(\frac{\partial V_{i}\left(t, \overline{\mathbf{v}}_{i}\right)}{\partial \mathbf{v}_{i}}\right)^{T} \\
\times\left[f_{i}\left(t, \mathbf{x}, \mathbf{v}_{i}(t, \mathbf{x})\right)+\sum_{j=1}^{N} H_{i j}\left(\mathbf{v}_{i}, \mathbf{v}_{j}, t\right)\right]
\end{gathered}
$$

$$
+\frac{1}{2} \operatorname{Trace}\left\{\left[g_{i}\left(t, \mathbf{x}, \mathbf{v}_{i}(t, \mathbf{x})\right)+\sum_{j=1}^{N} N_{i j}\left(\mathbf{v}_{i}, \mathbf{v}_{j}, t\right)\right]^{T}\right.
$$

$$
\begin{aligned}
& \times\left(V_{i}\left(t, \mathbf{v}_{i}\right)\right)_{\mathbf{v}_{i} \mathbf{v}_{j}}^{\prime \prime} \\
& \left.\times\left[g_{i}\left(t, \mathbf{x}, \mathbf{v}_{i}(t, \mathbf{x})\right)+\sum_{j=1}^{N} N_{i j}\left(\mathbf{v}_{i}, \mathbf{v}_{j}, t\right)\right]\right\},
\end{aligned}
$$

for $\forall t \geq 0$. Hence,

$$
\begin{aligned}
& V_{i}\left(t, \overline{\mathbf{v}}_{i}\left(t, t_{0}, \overline{\mathbf{v}_{\mathbf{i} \mathbf{0}}}\right)\right) \\
&=V_{i}\left(t_{0}, \overline{\mathbf{v}_{\mathbf{i} \mathbf{0}}}\right)+\int_{t_{0}}^{t} \mathscr{L} V_{i}\left(s, \overline{\mathbf{v}}_{i}\left(s, t_{0}, \overline{\mathbf{v}_{\mathbf{i} \mathbf{0}}}\right)\right) \mathrm{d} s \\
&+\int_{t_{0}}^{t} \frac{\partial V_{i}\left(s, \overline{\mathbf{v}}_{i}\right)}{\partial \overline{\mathbf{v}}_{i}} \int_{G}[ g_{i}\left(t, \mathbf{x}, \mathbf{v}_{i}(t, \mathbf{x})\right) \\
&\left.+\sum_{j=1}^{N} N_{i j}\left(\mathbf{v}_{i}, \mathbf{v}_{j}, t\right)\right] \mathrm{d} \mathbf{x} \mathrm{d} w(s) .
\end{aligned}
$$

Build a Lyapunov function with the form

$$
\bar{V}_{i}=e^{\widehat{b}\left(t-t_{0}\right)} V_{i}\left(t, \overline{\mathbf{v}}_{i}\right) .
$$

Applying Itô formula again, we obtain

$$
\begin{aligned}
d \bar{V}_{i}= & \widehat{b} e^{\hat{b}\left(t-t_{0}\right)} V_{i}\left(t, \overline{\mathbf{v}}_{i}\right) \mathrm{d} t+e^{\widehat{b}\left(t-t_{0}\right)} d V_{i} \\
= & e^{\widehat{b}\left(t-t_{0}\right)}\left[\widehat{b} V_{i}\left(t, \overline{\mathbf{v}}_{i}\right)+\mathscr{L} V_{i}\left(t, \overline{\mathbf{v}}_{i}\right)\right] \mathrm{d} t+e^{\widehat{b}\left(t-t_{0}\right)} \\
\times \int_{t_{0}}^{t} \frac{\partial V_{i}\left(s, \overline{\mathbf{v}}_{i}\right)}{\partial \overline{\mathbf{v}}_{i}} \int_{G} & {\left[g_{i}\left(t, \mathbf{x}, \mathbf{v}_{i}(t, \mathbf{x})\right)\right.} \\
& \left.+\sum_{j=1}^{N} N_{i j}\left(\mathbf{v}_{i}, \mathbf{v}_{j}, t\right)\right] \mathrm{d} \mathbf{x} \mathrm{d} w(t) .
\end{aligned}
$$

For any $n \geq\left|\mathbf{v}_{0}\right|_{G}$, define a stop-time

$$
\tau_{n}=\inf \left\{t \geq t_{0}:|\mathbf{v}(t, \mathbf{x})|_{G} \geq n\right\} .
$$


It follows from integrating (57) as to $t$ from $t_{0}$ to $t \wedge \tau_{n}$

$$
\begin{gathered}
e^{\widehat{b}\left(t \wedge \tau_{n}-t_{0}\right)} V_{i}\left(t \wedge \tau_{n}, \overline{\mathbf{v}}_{i}\left(t \wedge \tau_{n}\right)\right) \\
=V_{i}\left(t_{0}, \bar{v}_{0}\right)+\int_{t_{0}}^{t \wedge \tau_{n}} e^{\widehat{b}\left(s-t_{0}\right)}\left[\widehat{b} V_{i}\left(s, \overline{\mathbf{v}}_{i}\right)+\mathscr{L} V_{i}\left(s, \overline{\mathbf{v}}_{i}\right)\right] \mathrm{d} s \\
+\int_{t_{0}}^{t \wedge \tau_{n}} e^{\widehat{b}\left(s-t_{0}\right)} \frac{\partial V_{i}\left(s, \overline{\mathbf{v}}_{i}\right)}{\partial \overline{\mathbf{v}}_{i}} \\
\times \int_{G}\left[g_{i}\left(t, \mathbf{x}, \mathbf{v}_{i}(t, \mathbf{x})\right)\right. \\
\left.+\sum_{j=1}^{N} N_{i j}\left(\mathbf{v}_{i}, \mathbf{v}_{j}, t\right)\right] \mathrm{d} \mathbf{x} \mathrm{d} w(s) .
\end{gathered}
$$

As $\partial V_{i}\left(t, \overline{\mathbf{v}}_{i}\right) / \partial \overline{\mathbf{v}}_{i}$ is continuous on $R_{+} \times S_{h}$ and $g_{i}\left(t, \mathbf{x}, \mathbf{v}_{i}(t, \mathbf{x})\right)+\sum_{j=1}^{N} N_{i j}\left(\mathbf{v}_{i}, \mathbf{v}_{j}, t\right)$ satisfies integral linear growth condition, there must exist constant $L_{1}>0$ such that $\left|\partial V_{i}\left(t, \overline{\mathbf{v}}_{i}\right) / \partial \overline{\mathbf{v}}_{i}\right| \leq L_{1}$, so

$$
\begin{aligned}
& \left|\frac{\partial V_{i}\left(s, \overline{\mathbf{v}}_{i}\right)}{\partial \overline{\mathbf{v}}_{i}} \int_{G}\left[g_{i}\left(t, \mathbf{x}, \mathbf{v}_{i}(t, \mathbf{x})\right)+\sum_{j=1}^{N} N_{i j}\left(\mathbf{v}_{i}, \mathbf{v}_{j}, t\right)\right] \mathrm{d} \mathbf{x}\right| \\
& \quad \leq L_{1} K\left(1+\left|\int_{G} \mathbf{v}\left(t, \mathbf{x}, t_{0}, \mathbf{v}_{i 0}\right) \mathrm{d} \mathbf{x}\right|\right) \\
& \quad \leq L_{1} K(1+h) .
\end{aligned}
$$

By Theorem 2.8 of [35], we obtain

$$
\begin{aligned}
\mathscr{E}\left[\int_{t_{0}}^{t} \frac{\partial V_{i}\left(s, \overline{\mathbf{v}}_{i}\right)}{\partial \overline{\mathbf{v}}_{i}} \int_{G}[\right. & g_{i}\left(t, \mathbf{x}, \mathbf{v}_{i}(t, \mathbf{x})\right) \\
& \left.\left.+\sum_{j=1}^{N} N_{i j}\left(\mathbf{v}_{i}, \mathbf{v}_{j}, t\right)\right] \mathrm{d} \mathbf{x} \mathrm{d} w(s)\right]=0
\end{aligned}
$$

Thus, it can be derived from taking mathematical expectation at both sides of (59) that

$$
\begin{aligned}
\mathscr{E}\left\{e^{\widehat{b}\left(t \wedge \tau_{n}-t_{0}\right)} V_{i}\left(t \wedge \tau_{n}, \overline{\mathbf{v}}_{i}\left(t \wedge \tau_{n}\right)\right)\right\} \\
=V_{i}\left(t_{0}, \overline{v_{0}}\right) \\
\quad+\mathscr{E}\left\{\int_{t_{0}}^{t \wedge \tau_{n}} e^{\widehat{b}\left(s-t_{0}\right)}\left[\widehat{b} V_{i}\left(s, \overline{\mathbf{v}}_{i}\right)+\mathscr{L} V_{i}\left(s, \overline{\mathbf{v}}_{i}\right)\right] \mathrm{d} s\right\} .
\end{aligned}
$$

On the other hand, by (B5)(III), it is derived that

$$
\begin{aligned}
\mathscr{L} V(t, \overline{\mathbf{v}}) & =\sum_{i=1}^{N} c_{i} \mathscr{L} V_{i}\left(t, \overline{\mathbf{v}}_{i}\right) \\
& \leq-\sum_{i=1}^{N} c_{i} \widehat{b}_{i} V_{i}\left(t, \overline{\mathbf{v}}_{i}\right)+\sum_{i, j=1} c_{i} a_{i j} F_{i j}\left(\overline{\mathbf{v}}_{i}, \overline{\mathbf{v}}_{j}, t\right) .
\end{aligned}
$$

Making use of Lemma 5 with weighted digraph $(\mathscr{G}, A)$, it yields

$$
\begin{aligned}
& \sum_{i, j=1} c_{i} a_{i j} F_{i j}\left(\overline{\mathbf{v}}_{i}, \overline{\mathbf{v}}_{j}, t\right) \\
& =\sum_{\mathbb{Q} \in \mathbb{Q}} W(\mathscr{Q}) \sum_{(i, j) \in E\left(C_{Q}\right)} F_{i j}\left(\overline{\mathbf{v}}_{i}, \overline{\mathbf{v}}_{j}, t\right) .
\end{aligned}
$$

In view of Condition $(\mathrm{A} 2)$ and the fact that $W(Q)>0$, we get

$$
\begin{aligned}
\mathscr{L} V(t, \overline{\mathbf{v}}) \leq & -\sum_{i=1}^{N} c_{i} \widehat{b}_{i} V_{i}\left(t, \overline{\mathbf{v}}_{i}\right) \\
& +\sum_{Q \in \mathbb{Q}} W(\mathcal{Q}) \sum_{(i, j) \in E\left(C_{Q}\right)} F_{i j}\left(\overline{\mathbf{v}}_{i}, \overline{\mathbf{v}}_{j}, t\right) \\
\leq & -\sum_{i=1}^{N} c_{i} \widehat{b}_{i} V_{i}\left(t, \overline{\mathbf{v}}_{i}\right)<-\widehat{b} V(t, \overline{\mathbf{v}}) .
\end{aligned}
$$

Thus $V(t, \overline{\mathbf{v}})$ is a Lyapunov-B function for (12). Taking the mathematical expectation at the two sides of (59) and using (61), (63), and (65) and combining conditions (C1), (I), and (II), we have

$$
\begin{aligned}
\bar{c}_{1} e^{\widehat{b}\left(t \wedge \tau_{n}-t_{0}\right)} \mathscr{E}\left\{\left|\mathbf{y}\left(t \wedge \tau_{n}, \mathbf{x}\right)\right|_{G}\right\} \\
\quad \leq \mathscr{E}\left\{e^{\widehat{b}\left(t \wedge \tau_{n}-t_{0}\right)} V\left(t \wedge \tau_{n}, \overline{\mathbf{v}}\right)\right\} \leq V\left(t_{0}, \overline{\mathbf{v}_{0}}\right) .
\end{aligned}
$$

Obviously $\tau_{n} \rightarrow \infty$ when $n \rightarrow \infty$, we have

$$
\bar{c}_{1} e^{\widehat{b}\left(t-t_{0}\right)} \mathscr{E}\left\{|\mathbf{y}(t, \mathbf{x})|_{G}\right\} \leq \bar{c}_{2}\left(\left|\mathbf{v}_{0}\right|_{G}\right)
$$

Therefore, we get

$$
\mathscr{E}\left\{|\mathbf{y}(t, \mathbf{x})|_{G}\right\} \leq \frac{\bar{c}_{2}}{\bar{c}_{1}}\left|v_{0}\right|_{G} e^{-\widehat{b}\left(t-t_{0}\right)}, \quad t \geq 0,
$$

as required.

According to Theorem 18, it is easy to reach the following theorem.

Theorem 19. Let $\overline{\mathbf{v}}_{i}(t)=\int_{G} \mathbf{v}_{i}(t, \mathbf{x}) d \mathbf{x}$. Suppose that the following conditions hold.

(C1) There exist positive-definite functions $V_{i}(t, \xi) \quad \epsilon$ $C^{1,2}\left(\mathbb{R}_{+} \times \mathbb{R}^{n_{i}} ; \mathbb{R}_{+}\right)$, functions $F_{i j}\left(\overline{\mathbf{v}}_{i}, \overline{\mathbf{v}}_{j}, t\right)$, and constants $a_{i j} \geq 0$ satisfying the following.

(I) $V_{i}\left(t, \overline{\mathbf{v}}_{i}(t)\right) \in C^{1,2}\left[\mathbb{R}_{+} \times S_{h}, \mathbb{R}\right],\left(S_{h}=\{\zeta \mid\right.$ $|\zeta(\cdot)|<h\}$ ). (Here, function $V_{i}\left(t, \overline{\mathbf{v}}_{i}(t)\right)$ gets rid of the restriction of $\left.V_{i}(t, 0)=0\right)$.

(II) $\widehat{c}_{1}|\mathbf{y}(t, \mathbf{x})|_{G} \leq V\left(t, \overline{\mathbf{v}}(t) \leq \widehat{c}_{2}|\mathbf{v}(t, \mathbf{x})|_{G}\right.$, where $V(t, \overline{\mathbf{v}}) \triangleq \sum_{i=1}^{N} c_{i} V_{i}\left(t, \overline{\mathbf{v}}_{i}\right), c_{i}$ is defined as (8), and $\widehat{c}_{1}$ and $\widehat{c}_{2}<1$ are positive constants.

(III) $\mathscr{L} V_{i}\left(t, \overline{\mathbf{v}}_{i}\right) \leq-\widehat{b}_{i} V_{i}\left(t, \overline{\mathbf{v}}_{i}\right)+\sum_{j=1}^{n} a_{i j} F_{i j}\left(\overline{\mathbf{v}}_{i}, \overline{\mathbf{v}}_{j}, t\right)$, constants $\widehat{b}_{i}>0, \quad t \geq 0, i=1,2, \ldots, N$.

(C2) Condition (A2) holds, if $(\mathscr{G}, A)$ is balanced and (29) holds. 
Then, function $V(t, \overline{\mathbf{v}}) \triangleq \sum_{i=1}^{N} c_{i} V_{i}\left(t, \overline{\mathbf{v}}_{i}\right)$ is a Lyapunov- $A$ function for (12), and

$$
E\left\{|\mathbf{y}(t, \mathbf{x})|_{G}\right\} \leq \frac{\bar{c}_{2}}{\bar{c}_{1}}\left|v_{0}\right|_{G} e^{-\widehat{b}\left(t-t_{0}\right)}, \quad t \geq t_{0},
$$

where $\widehat{b}=\min \left\{\widehat{b}_{1}, \ldots, \widehat{b}_{N}\right\}$; that is, the trivial solution of system (12) is exponentially stable in mean as to partial variables $\mathbf{y}$.

Remark 20. In this section, we derive some novel findings on stability principles for uniform stability in mean, asymptotic stability in mean, uniformly asymptotic stability in mean, and exponential stability in mean of partial variables for CSRDSNs. The results in previous literature [38] are special cases of our findings, because our results have a close relation to the topology property of the network. When we employ Lyapunov function method to tackle the stability problems for coupled stochastic large-scale systems, the most difficult thing is to construct a Lyapunov function. We also provide a systematic method for constructing a global Lyapunov function for these CSRDSNs by using graph theory. The new method is helpful to analyze the dynamics of complex networks.

\section{Example}

Consider the 2-dimensional Itô SRDSMS (1) satisfying the bounded condition (2), and we assume $(\mathscr{G}, A)$ is strongly connected and balanced. Consider

$$
\begin{aligned}
& \mathrm{d} \mathbf{v}_{1}(t, \mathbf{x}) \\
& =\left[\Delta \mathbf{v}_{1}(t, \mathbf{x})+\mathbf{v}_{2}(t, \mathbf{x})-\sum_{j=1}^{2} a_{1 j}\left(\mathbf{v}_{1}(t, \mathbf{x})-\mathbf{v}_{j}(t, \mathbf{x})\right)\right] \mathrm{d} t \\
& \mathrm{~d} \mathbf{v}_{2}(t, \mathbf{x}) \\
& =\left[\Delta \mathbf{v}_{2}(t, \mathbf{x})-\mathbf{v}_{1}(t, \mathbf{x})-\alpha(t) \mathbf{v}_{2}(t, \mathbf{x})\right. \\
& \left.\quad+\sum_{j=1}^{2} a_{2 j}\left(\mathbf{v}_{2}(t, \mathbf{x})-\mathbf{v}_{j}(t, \mathbf{x})\right)\right] \mathrm{d} t \\
& \quad-\sqrt{\alpha(t)} \mathbf{v}_{2}(t, \mathbf{x}) \mathrm{d} W(t) .
\end{aligned}
$$

Construct function $V=\left(\int_{G} \mathbf{v}_{1}(t, \mathbf{x}) \mathrm{d} \mathbf{x}\right)^{2}+\left(\int_{G} \mathbf{v}_{2}(t, \mathbf{x}) \mathrm{d} \mathbf{x}\right)^{2}$, and we have

$$
\begin{aligned}
\mathscr{L} V= & \left(2 \int_{G} \mathbf{v}_{1} \mathrm{~d} \mathbf{x} 2 \int_{G} \mathbf{v}_{2} \mathrm{~d} \mathbf{x}\right)\left(\begin{array}{l}
\int_{G} \mathbf{v}_{2} \mathrm{~d} \mathbf{x} \\
-\int_{G} \mathbf{v}_{1} \mathrm{~d} \mathbf{x}-\alpha(t) \int_{G} \mathbf{v}_{2} \mathrm{~d} \mathbf{x}
\end{array}\right) \\
& +\alpha(t)\left(\int_{G} \mathbf{v}_{2}(t, \mathbf{x}) \mathrm{d} \mathbf{x}\right)^{2}+\sum_{j=1}^{2} a_{k j} F_{k j}\left(\mathbf{v}_{k}, \mathbf{v}_{j}, t\right) \\
= & -\alpha(t)\left(\int_{G} \mathbf{v}_{2}(t, \mathbf{x}) \mathrm{d} \mathbf{x}\right)^{2}<0 .
\end{aligned}
$$

According to Theorem 6, we know the trial solution of system (70) is stable in mean as to partial variable $\mathbf{v}_{2}$.

\section{Conclusions}

In this paper, stability in mean of partial variables for coupled stochastic reaction-diffusion systems on networks (CSRDSNs) is considered. By transforming the integral of the trajectory with respect to spatial variables as the solution of the stochastic ordinary differential equations (SODE) and using Itô formula, some novel stability principles are established for uniform stability in mean, asymptotic stability in mean, uniformly asymptotic stability in mean, and exponential stability in mean of partial variables for CSRDSNs. These stability principles have a close relation with the topology property of the network. A systematic method for constructing global Lyapunov function for these CSRDSNs is also provided by using graph theory. Our methods can be extended to deal with coupled stochastic neutral differential equations on networks. Future work is to give a systematic approach to build a Lyapunov functional for coupled Markovian switching reaction-diffusion systems on networks.

\section{Conflict of Interests}

The authors declare that there is no conflict of interests regarding the publication of this paper.

\section{Acknowledgments}

This research is supported by the Natural Science Foundation of Guangxi Autonomous Region (nos. 2012GXNSFBA053003, 2013YB141), the National Natural Science Foundations of China (60973048, 61272077, 60974025, 60673101, and 60939003), National 863 Plan Project (2008 AA04Z401, 2009AA043404), the Natural Science Foundation of Shandong Province (no. ZR2012FM006), the Natural Scientific Research Innovation Foundation in Harbin Institute of Technology (HIT.NSRIF. 2001120), and by the Polish-Norwegian Research Programme operated by the National Centre for Research and 24 Development under the Norwegian Financial Mechanism 2009-2014 in the frame of Project Contract no. PolNor/200957/47/2013.

\section{References}

[1] S. H. Strogatz, "Exploring complex networks," Nature, vol. 410, no. 6825 , pp. 268-276, 2001.

[2] D. J. Watts and S. H. Strogatz, "Collective dynamics of smallworld networks," Nature, vol. 393, no. 6684, pp. 440-442, 1998.

[3] R. Pastor-Satorras and A. Vespignani, "Epidemic dynamics and endemic states in complex networks," Physical Review E: Statistical, Nonlinear, and Soft Matter Physics, vol. 63, no. 6, Article ID 066117, pp. 1-8, 2001.

[4] J. Lu, D. W. C. Ho, J. Cao, and J. Kurths, "Exponential synchronization of linearly coupled neural networks with impulsive 
disturbances," IEEE Transactions on Neural Networks, vol. 22, no. 2, pp. 329-335, 2011.

[5] J. Lu, D. W. C. Ho, and J. Cao, "A unified synchronization criterion for impulsive dynamical networks," Automatica, vol. 46, no. 7, pp. 1215-1221, 2010.

[6] W. Yu, J. Cao, and W. Lu, "Synchronization control of switched linearly coupled neural networks with delay," Neurocomputing, vol. 73, no. 4-6, pp. 858-866, 2010.

[7] S. Boccaletti, V. Latora, Y. Moreno, M. Chavez, and D.-U. Hwang, "Complex networks: structure and dynamics," Physics Reports, vol. 424, no. 4-5, pp. 175-308, 2006.

[8] A. E. Motter, C. Zhou, and J. Kurths, "Network synchronization, diffusion, and the paradox of heterogeneity," Physical Review E: Statistical, Nonlinear, and Soft Matter Physics, vol. 71, no. 1, Article ID 016116, 2005.

[9] T. Nishikawa, A. E. Motter, Y. Lai, and F. C. Hoppensteadt, "Heterogeneity in oscillator networks: are smaller worlds easier to synchronize?” Physical Review Letters, vol. 91, no. 1, Article ID 014101, pp. 1-4, 2003.

[10] C. W. Wu, "On the relationship between pinning control effectiveness and graph topology in complex networks of dynamical systems," Chaos, vol. 18, no. 3, Article ID 037103, 2008.

[11] M. Y. Li and Z. Shuai, "Global-stability problem for coupled systems of differential equations on networks," Journal of Differential Equations, vol. 248, no. 1, pp. 1-20, 2010.

[12] Z.-R. Xiang, C.-H. Qiao, and M. S. Mahmoud, "Finite-time analysis and $H_{\infty}$ control for switched stochastic systems," Journal of the Franklin Institute, vol. 349, no. 3, pp. 915-927, 2012.

[13] G.-X. Chen, Z.-R. Xiang, and M. S. Mahmoud, "Stability and $H_{\infty}$ performance analysis of switched stochastic neutral systems," Circuits, Systems, and Signal Processing, vol. 32, no. 1, pp. 387-400, 2013.

[14] Y. G. Kao, H. Sun, and H. X. Cao, "Stability analysis for coupled stochastic systems with time delay on networks," Mathematica Applicata, vol. 26, pp. 67-75, 2013.

[15] Y. Kao, C. Wang, and L. Zhang, "Delay-dependent exponential stability of impulsive Markovian jumping cohen-grossberg neural networks with reaction-diffusion and mixed delays," Neural Processing Letters, vol. 38, no. 3, pp. 321-346, 2013.

[16] C. H. Wang, Y. G. Kao, and G. Yang, "Exponential stability of impulsive stochastic fuzzy reaction-diffusion Cohen-Grossberg neural networks with mixed delays," Neurocomputing, vol. 89, pp. 55-63, 2012.

[17] Y. G. Kao, C. H. Wang, H. R. Hamid, and R. Bi, “Global stability of coupled Markovian switching reaction-diffusion systems on networks," Nonlinear Analysis: Hybrid Systems, 2014.

[18] Q. Luo and Y. Zhang, "Almost sure exponential stability of stochastic reaction diffusion systems," Nonlinear Analysis, Theory, Methods and Applications, vol. 71, no. 12, pp. e487-e493, 2009.

[19] L. Qi, F. Deng, J. Bao, B. Zhao, and Y. Fu, "Stabilization of stochastic Hopfield neural network with distributed parameters," Science in China, Series F: Information Sciences, vol. 47, no. 6, pp. 752-762, 2004.

[20] Y. Kao, J. Guo, C. Wang, and X. Sun, "Delay-dependent robust exponential stability of Markovian jumping reaction-diffusion Cohen-Grossberg neural networks with mixed delays," Journal of The Franklin Institute, vol. 349, no. 6, pp. 1972-1988, 2012.

[21] Y. G. Kao and C. H. Wang, "Global stability analysis for stochastic coupled reaction-diffusion systems on networks," Nonlinear Analysis B: Real World Applications, vol. 14, no. 3, pp. 1457-1465, 2013.
[22] J. Hu, Z.-D. Wang, and B. Shen, "Quantized filtering for a class of nonlinear systems with multiplicative noises and missing measurements," International Journal of Control, vol. 86, no. 4, pp. 650-663, 2013.

[23] J. Hu, D.-Y. Chen, and J.-H. Du, "State estimation for a class discrete nonlinear systems with randomly occurring uncertainties and distributed sensor delays," International Journal of General Systems, vol. 43, pp. 387-401, 2014.

[24] J. Hu, Z.-D. Wang, and H.-L. Dong, "Recent advances on recursive filtering and sliding mode design for networked nonlinear stochastic systems: a survey," Mathematical Problems in Engineering, vol. 2013, Article ID 646059, 12 pages, 2013.

[25] J. Hu, Z.-D. Wang, and H.-J. Gao, "Recursive filtering wit random parameter matrices, multiple fading measurements and correlated noises," Automatica, vol. 49, no. 11, pp. 34403448, 2013.

[26] Z.-R. Xiang, C.-H. Qiao, and M. S. Mahmoud, "Robust $H_{\infty}$ filtering for switched stochastic systems under asynchronous switching," Journal of the Franklin Institute, vol. 349, no. 3, pp. 1213-1230, 2012.

[27] Y. Kao, C. Wang, F. Zha, and H. Cao, "Stability in mean of partial variables for stochastic reaction-diffusion systems with Markovian switching," Journal of the Franklin Institute, vol. 351, no. 1, pp. 500-512, 2014.

[28] Rumiantsev, V. Vitalevich, Oziraner, and A. Solomonovich, Stability and Stabilization of Motion with Respect to Part of the Variables (in Russian), Izdatel'stvo Nauka, Moscow, Russia, 1987.

[29] V. I. Vorotnikov, Partial Stability and Control, Birkhauser, Boston, Mass, USA, 1998.

[30] S. Ibrir, "Static output feedback and guaranteed cost control of a class of discrete-time nonlinear systems with partial state measurements," Nonlinear Analysis, Theory, Methods and Applications, vol. 68, no. 7, pp. 1784-1792, 2008.

[31] J. Xi, Z. Shi, and Y. Zhong, "Output consensus analysis and design for high-order linear swarm systems: partial stability method," Automatica, vol. 48, no. 9, pp. 2335-2343, 2012.

[32] M. H. Shafiei and T. Binazadeh, "Partial stabilization-based guidance," ISA Transactions, vol. 51, no. 1, pp. 141-145, 2012.

[33] X. Dai, "Weakly Birkhoff recurrent switching signals, almost sure and partial stability of linear switched dynamical systems," Journal of Differential Equations, vol. 250, no. 9, pp. 3584-3629, 2011.

[34] O. Ignatyev, "Partial asymptotic stability in probability of stochastic differential equations," Statistics and Probability Letters, vol. 79, no. 5, pp. 597-601, 2009.

[35] T. Binazadeh and M. J. Yazdanpanah, "Partial stabilization of uncertain nonlinear systems," ISA Transactions, vol. 51, no. 2, pp. 298-303, 2012.

[36] W.-H. Chen, X. Lu, and F. Chen, "Impulsive synchronization of chaotic Lur'e systems via partial states," Physics Letters A: General, Atomic and Solid State Physics, vol. 372, no. 23, pp. 4210-4216, 2008.

[37] V. I. Vorotnikov and Y. G. Martyshenko, "On partial detectability of the nonlinear dynamic systems," Automation and Remote Control, vol. 70, no. 1, pp. 20-32, 2009.

[38] Y. Zhang, Q. Luo, and X. Lai, "Stability in mean of partial variables for stochastic reaction diffusion systems," Nonlinear Analysis, Theory, Methods and Applications, vol. 71, no. 12, pp. e550-e559, 2009.

[39] X. Mao, Exponential Stability of Stochastic Differential Equations, Marcel Dekker, New York, NY, USA, 1994. 
[40] X. Mao, Stochastic Differential Equations and Their Applications, Horwood Publishing, Chichester, UK, 1997.

[41] D. B. West, Introduction to Graph Theory, Prentice Hall, Upper Saddle River, NJ, USA, 1996.

[42] A. Friedman, Stochastic Differential Equations and Applications, vol. 1, Academic Press, New York, NY, USA, 1975.

[43] A. Friedman, Stochastic Differential Equations and Applications, vol. 2, Academic Press, New York, NY, USA, 1983. 


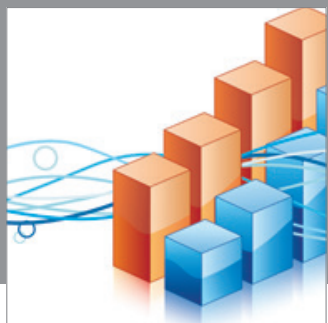

Advances in

Operations Research

mansans

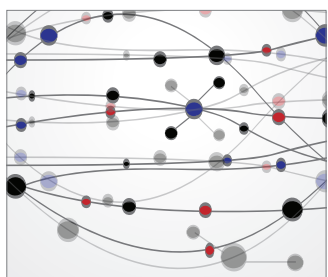

The Scientific World Journal
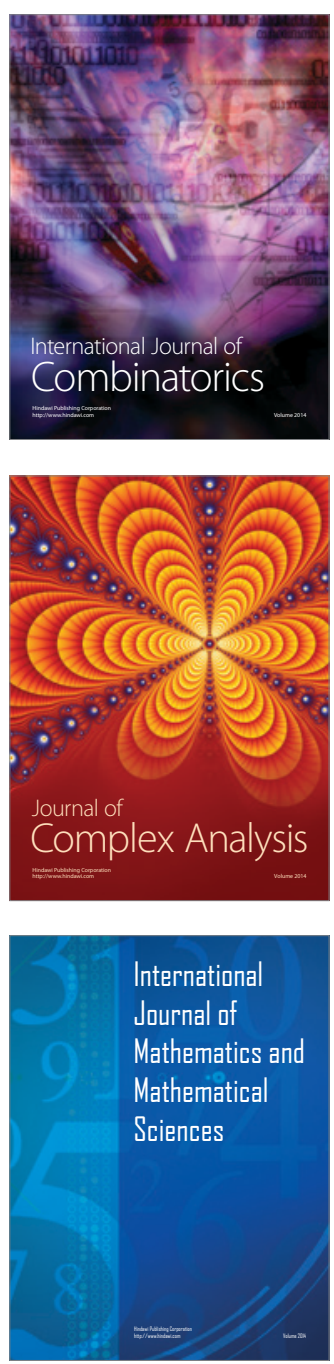
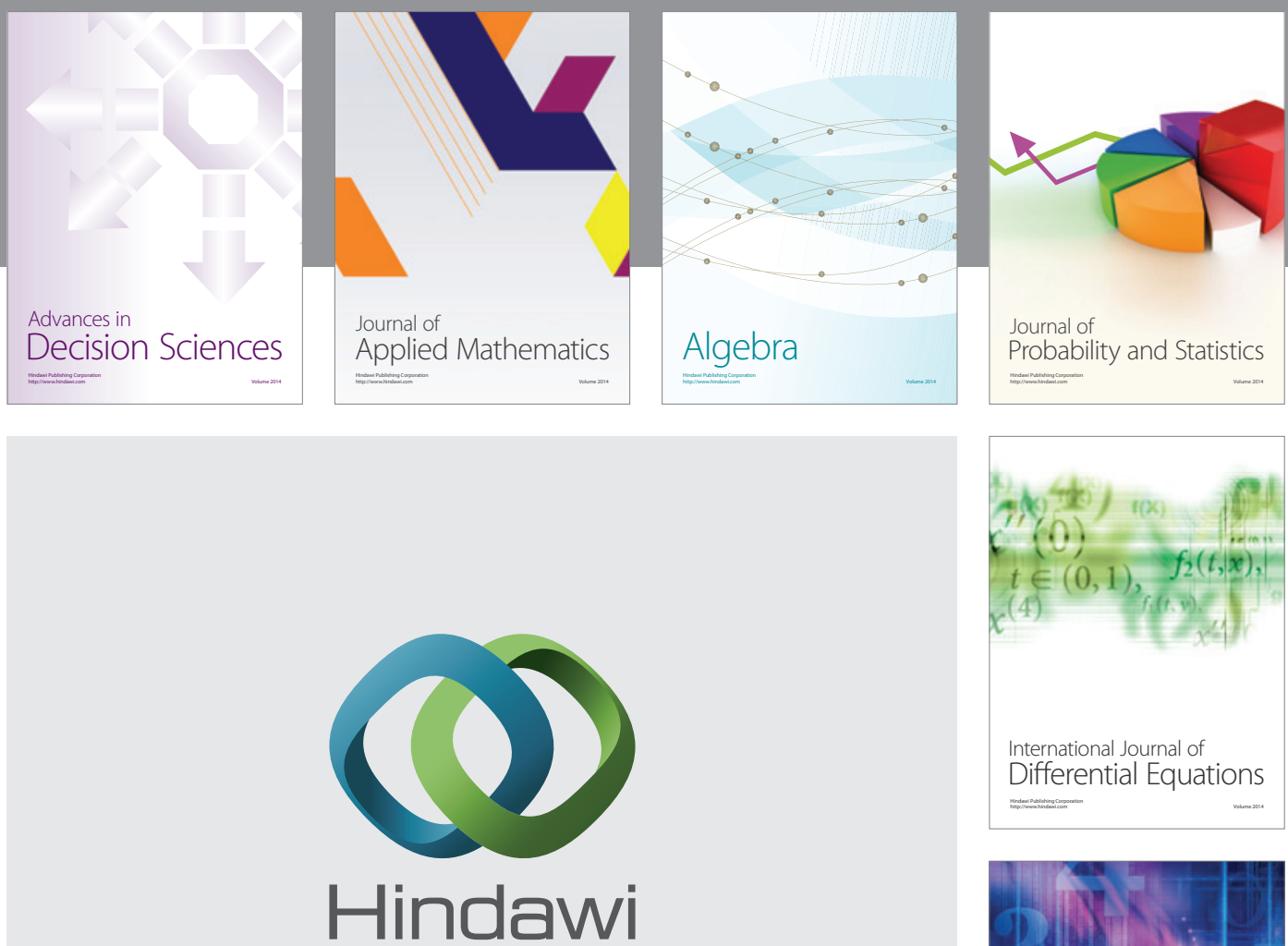

Submit your manuscripts at http://www.hindawi.com
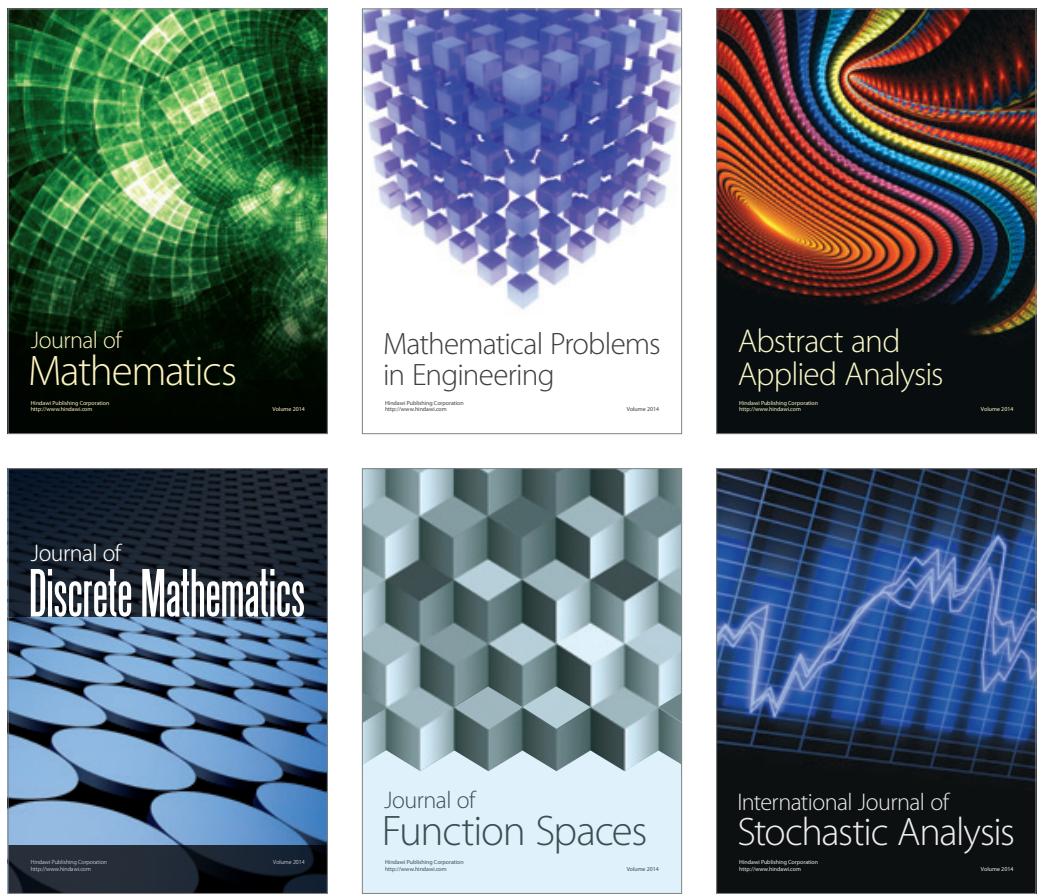

Journal of

Function Spaces

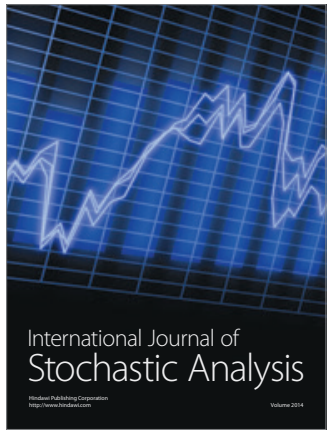

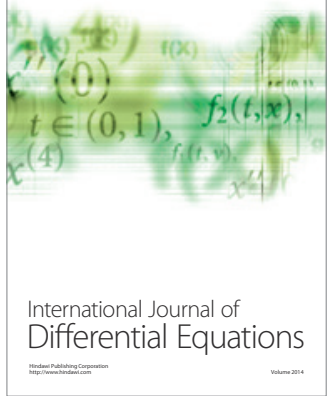
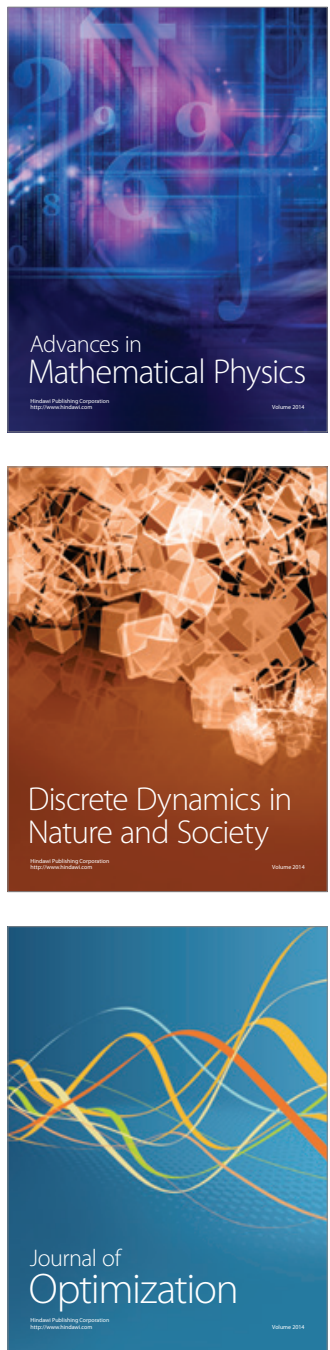